\title{
Motor Patterns During Walking on a Slippery \\ Walkway
}

Germana Cappellini, Yuri P. Ivanenko, Nadia Dominici, Richard E. Poppele and Francesco Lacquaniti

J Neurophysiol 103:746-760, 2010. First published 2 December 2009; doi:10.1152/jn.00499.2009

You might find this additional info useful...

This article cites 81 articles, 27 of which can be accessed free at:

http://jn.physiology.org/content/103/2/746.full.html\#ref-list-1

This article has been cited by 3 other HighWire hosted articles

Slipping, sliding and stability: locomotor strategies for overcoming low-friction surfaces

Andrew J. Clark and Timothy E. Higham

J Exp Biol, April 15, 2011; 214 (8): 1369-1378.

[Abstract] [Full Text] [PDF]

\author{
Migration of Motor Pool Activity in the Spinal Cord Reflects Body Mechanics in Human \\ Locomotion \\ Germana Cappellini, Yuri P. Ivanenko, Nadia Dominici, Richard E. Poppele and Francesco \\ Lacquaniti \\ J Neurophysiol, December , 2010; 104 (6): 3064-3073. \\ [Abstract] [Full Text] [PDF] \\ Age-Related Modifications of Muscle Synergies and Spinal Cord Activity During \\ Locomotion \\ Vito Monaco, Alessio Ghionzoli and Silvestro Micera \\ J Neurophysiol, October , 2010; 104 (4): 2092-2102. \\ [Abstract] [Full Text] [PDF]
}

Updated information and services including high resolution figures, can be found at:

http://jn.physiology.org/content/103/2/746.full.html

Additional material and information about Journal of Neurophysiology can be found at: http://www.the-aps.org/publications/jn

This infomation is current as of November 24, 2011.

Journal of Neurophysiology publishes original articles on the function of the nervous system. It is published 12 times a year (monthly) by the American Physiological Society, 9650 Rockville Pike, Bethesda MD 20814-3991. Copyright @ 2010 by the American Physiological Society. ISSN: 0022-3077, ESSN: 1522-1598. Visit our website at http://www.the-aps.org/. 


\title{
Motor Patterns During Walking on a Slippery Walkway
}

\author{
Germana Cappellini, ${ }^{1}$ Yuri P. Ivanenko, ${ }^{1}$ Nadia Dominici, ${ }^{1,2}$ Richard E. Poppele, ${ }^{4}$ and Francesco Lacquaniti ${ }^{1,2,3}$ \\ ${ }^{1}$ Laboratory of Neuromotor Physiology, Santa Lucia Foundation; ${ }^{2}$ Centre of Space Biomedicine and ${ }^{3}$ Department of Neuroscience, \\ University of Rome Tor Vergata, Rome, Italy; and ${ }^{4}$ Department of Neuroscience, University of Minnesota, Minneapolis, Minnesota
}

Submitted 8 June 2009; accepted in final form 30 November 2009

Cappellini G, Ivanenko YP, Dominici N, Poppele RE, Lacquaniti F. Motor patterns during walking on a slippery walkway. J Neurophysiol 103: 746-760, 2010. First published December 2, 2009; doi:10.1152/jn.00499.2009. Friction and gravity represent two basic physical constraints of terrestrial locomotion that affect both motor patterns and the biomechanics of bipedal gait. To provide insights into the spatiotemporal organization of the motor output in connection with ground contact forces, we studied adaptation of human gait to steady low-friction conditions. Subjects walked along a slippery walkway ( $7 \mathrm{~m}$ long; friction coefficient $\simeq 0.06$ ) or a normal, nonslippery floor at a natural speed. We recorded gait kinematics, ground reaction forces, and bilateral electromyographic (EMG) activity of 16 leg and trunk muscles and we mapped the recorded EMG patterns onto the spinal cord in approximate rostrocaudal locations of the motoneuron $(\mathrm{MN})$ pools to characterize the spatiotemporal organization of the motor output. The results revealed several idiosyncratic features of walking on the slippery surface. The step length, cycle duration, and horizontal shear forces were significantly smaller, the head orientation tended to be stabilized in space, whereas arm movements, trunk rotations, and lateral trunk inclinations considerably increased and foot motion and gait kinematics resembled those of a nonplantigrade gait. Furthermore, walking on the slippery surface required stabilization of the hip and of the center-of-body mass in the frontal plane, which significantly improved with practice. Motor patterns were characterized by an enhanced (roughly twofold) level of MN activity, substantial decoupling of anatomical synergists, and the absence of systematic displacements of the center of MN activity in the lumbosacral enlargement. Overall, the results show that when subjects are confronted with unsteady surface conditions, like the slippery floor, they adopt a gait mode that tends to keep the COM centered over the supporting limbs and to increase limb stiffness. We suggest that this behavior may represent a distinct gait mode that is particularly suited to uncertain surface conditions in general.

\section{N T R O D U C T I O N}

The nervous system has been considered to play a primary role in the control of various modes of locomotion. It is important to realize, however, that the control is coupled to the biomechanics of limb motion and an understanding of control strategies must also include the mechanical system. Accordingly, the optimization of human gaits (Saibene and Minetti 2003; Srinivasan and Ruina 2006) and the actual output of central pattern generators (CPGs; Grillner 1981) may be directly related to the biomechanics of limb loading. On Earth, our body is evolutionarily adapted to typical environmental surfaces where the friction coefficient is sufficiently high to provide efficient and secure gait. This raises the question about

Address for reprint requests and other correspondence: G. Cappellini, Laboratory of Neuromotor Physiology, Scientific Institute Foundation Santa Lucia, 306 via Ardeatina, 00179 Rome, Italy (E-mail: g.cappellini@ hsantalucia.it). how the system might respond to a different set of environmental conditions, such as low-friction conditions.

The mechanisms and adaptation to low friction represent a challenging area of research, both from a theoretical standpoint (e.g., the role of friction for the control of biped robots; Kajita et al. 2004; Park and Kwon 2001) and in the context of preventing potential falls and injury (Redfern et al. 2001). Various studies have focused on the adaptation to slip exposure for a single step in the middle of a nonslippery walkway. Furthermore, most studies (however, see Troy and Grabiner 2007) dealt with sagittal plane adjustments to slippery perturbations (Bhatt and Pai 2008; Chambers and Cham 2007; Heiden et al. 2006; Moyer et al. 2009; Troy et al. 2009; You et al. 2001) or to an anterior slip (Marigold and Patla 2002; Tang et al. 1998; Yang et al. 2008). These studies have shown that the exact values for detailed slip categorization depend on the frictional property of the interface materials, walking conditions, and biomechanics (e.g., walking speed, leg stiffness, foot kinematics, and the center-of-pressure [COP] at heel contact, etc.) (Redfern et al. 2001). Microslips $(<1 \mathrm{~cm})$ at heel contact naturally occur during most steps on dry, nonslippery surfaces (Perkins 1978; Strandberg and Lanshammar 1981). These slips occur without the knowledge of the walker who becomes aware of them only as the slipping distance is increased. A slipping distance of about $10-20 \mathrm{~cm}$ may be the threshold for a fall in adult subjects (Brady et al. 2000; Strandberg and Lanshammur 1981), although this distance and the recovery rate are reduced among older adults (Lockhart and Kim 2006). The shear to normal force ratio has been interpreted as the frictional requirement for a slip outcome. For soapy conditions on level surfaces, this ratio was reported to be as low as 0.02 (a fall case) $\leq 0.15$ (mini-stick), compared with about 0.2 for grip trials (Grönqvist et al. 1993; Strandberg 1983).

Although it is logical to postulate that human responses to slip perturbations result from multisensory (proprioceptive, vestibular, and visual) control of balance and locomotor pattern, nevertheless a specific role might be assigned to individual friction-related sensory pathways. For instance, cats with unilateral section of the dorsal spinocerebellar tract can walk normally at different speeds and perform various locomotor and postural tasks (limb loading, gait initiation, jumping from a chair, walking on a narrow beam, etc.). The only task that the affected limb cannot perform is walking on a slippery floor (R. E. Poppele, unpublished observation). In the latter case, the limb slips over the support surface, whereas the nonaffected limbs adapt immediately to altered contact forces and do not slip. This anecdotal observation indicates that appropriate horizontal shear forces are not a simple consequence of limb loading or pattern generation, but may have a specific repre- 
sentation and control by the CNS. Taking into account that the dorsal spinocerebellar tract provides information to the cerebellum about both foot kinematics and kinetics (Bosco et al. 2006; Poppele and Bosco 2003), a lack of this information may be critical for the control of shear forces in the slippery-surface gait.

There are at least two major mechanisms of adjustment that could occur during walking on the slippery surface. One is a step-by-step modification or optimization of a normal gait pattern that might involve various sensory mechanisms and the other is adoption of an appropriate intrinsic strategy already present in the repertoire of normal human gaits. In the latter case, subjects may adopt a specific strategy for walking on slippery surfaces in the form of a separate gait or mode of locomotion. Gaits are typically classified in terms of mechanics and organization of the motor output (Full and Koditschek 1999; Golubitsky et al. 1999; Grillner 1981; Ivanenko et al. 2008). A gait has been defined as "a pattern of locomotion characteristic of a limited range of speeds described by quantities of which one or more change discontinuously at transitions to other gaits" (Alexander 1989). Thus one way to distinguish between adaptations to a slippery surface and the adoption of a possible specific gait is to determine whether there is an abrupt transition to a different locomotion pattern that continues on a slippery surface. We investigated this by having subjects walk on a slippery walkway whose friction was similar to that of a natural icy surface (see also Fong et al. 2008a,b; Menant et al. 2009). In particular, we hypothesized that slippery-surface walking takes place with a unique and specific gait rather than a continuous series of adaptations during otherwise normal walking.

To test this hypothesis, we examined whether the main features of walking on a slippery surface develop with experience on the surface or whether they are already present in the first trial. We studied various biomechanical and electromyographic characteristics that are typically used for gait classifications: foot and trunk motion (Full and Koditschek 1999), ground reaction forces (Kuo et al. 2005), the center-of-body mass (COM; Margaria 1976), covariation of angular segment motion (Ivanenko et al. 2007), and spatiotemporal organization of the motor output (Ivanenko et al. 2008).

\section{METHODS}

\section{Participants}

Participants were six healthy volunteers (four males, two females; mean age $33 \pm 12 \mathrm{yr}$ [mean $\pm \mathrm{SD}$ ], height $175 \pm 11 \mathrm{~cm}$, and weight $73 \pm 12 \mathrm{~kg}$ ). None of the subjects had any history of neurological or orthopedic disease. Informed consent was obtained from all participants. The experiments were performed according to the procedures of the Ethics Committee of the Santa Lucia Institute and in conformity with the declaration of Helsinki for experiments on humans.

\section{Procedures}

Two main walking conditions were studied. First, a baseline measure was collected to study normal gait prior to any exposure to slipping (protocol 1) and then walking on the slippery surface was recorded (protocol 2).

PROTOCOL 1: WALKING ON THE NONSLIPPERY FLOOR. Walking on the nonslippery floor (7-m walkway) was recorded at different speeds to match walking speed conditions, since we expected that walking on the slippery surface would be slower than walking on the dry floor at a natural speed. On verbal instruction prior to start, subjects had to walk adopting what they considered a "very slow pace," "slow pace," or "normal pace." On average, 15 trials were recorded. To measure ground reaction forces and calculate joint torques, an appropriate initial position was chosen so that subjects stepped on the force plate with the right foot, although no particular task was given to the subject regarding foot placements. Subjects were asked to look straight ahead and to swing their arms as they would spontaneously do when walking with no particular purpose.

PROTOCOL 2: WALKING ON THE SLIPPERY SURFACE. Subjects were asked to walk on the slippery surface $(7 \times 1 \mathrm{~m}$ [length $\times$ width], using oil; the floor and the force plate were protected by a polyethylene pellicle, $0.07-\mathrm{mm}$ thickness) at a natural speed. For the slippery condition, oil was uniformly applied across the entire surface of the polyethylene film coating floor sample, so that it formed a thin layer on the $1 \times 7-m$ walkway without spilling off the walking path. Participants wore shoe covers over their bare feet that were made from the same thin polypropylene material used for the floor to reduce the interface friction. The operational dynamic coefficient of friction during walking on the slippery surface estimated as the mean ratio of the shear to normal foot force (Heiden et al. 2006) during late stance of the gait cycle (measured when the foot slightly slipped in the lateral direction; see RESULTS) was $0.06 \pm 0.1$ (mean \pm SD). A research assistant always walked (on the nonslippery floor) beside the subject to catch him/her in case of a fall. In the middle of the walkway subjects stepped on the force plate. The measured ground reaction shear forces were not significantly affected by the presence of the polyfilm covering the force plate. No practice was allowed prior to the recordings. Thirteen consecutive trials of walking on the slippery surface were recorded in each subject.

Protocols 1 and 2, each lasting about 30-40 min, were performed in one session with an approximately 30 -min rest between protocols. In the additional experimental session (on a separate day) we also recorded 10 overground running trials along a $10-\mathrm{m}$ walkway at a natural speed to compare the general spatiotemporal characteristics of the motor patterns across gaits.

\section{Data recording}

Kinematic data were recorded bilaterally at $100 \mathrm{~Hz}$ by means of the Vicon-612 system (Oxford, UK). Nine TV cameras were spaced around the walkway. The spatial accuracy of the system is $>1 \mathrm{~mm}$ (root mean square). Infrared reflective markers (diameter: $1.4 \mathrm{~cm}$ ) were attached on each side of the subject to the skin overlying the following landmarks: gleno-humeral joint (GH), elbow (Elb), wrist (Wri), ilium (IL), greater trochanter (GT), lateral femur epicondyle (LE), lateral malleolus (LM), heel (HE), and fifth metatarso-phalangeal joint (VM). In addition, we attached three markers to the head (two anterior markers on the forehead and one posterior marker on the back of the head).

The ground reaction forces $\left(\mathrm{F}_{\mathrm{x}}, \mathrm{F}_{\mathrm{y}}\right.$, and $\left.\mathrm{F}_{\mathrm{z}}\right)$ were recorded at $1,000 \mathrm{~Hz}$ by a force platform $(0.9 \times 0.6 \mathrm{~m}$; Kistler 9287B, Zurich, Switzerland $)$.

Electromyographic (EMG) activity was recorded by means of surface electrodes from 32 muscles simultaneously. The following 16 muscles were recorded from each body side: tibialis anterior (TA), gastrocnemius lateralis (LG), gastrocnemius medialis (MG), soleus (Sol), peroneus longus (PERL), vastus lateralis (Vlat), vastus medialis (Vmed), rectus femoris (RF), sartorius (SART), biceps femoris (long head, BF), semitendinosus (ST), adductor longus (ADD), tensor fascia latae (TFL), gluteus maximus (GM), gluteus medius (Gmed), and erector spinae, recorded at L2 (ESL2). The activity was recorded using active Delsys electrodes (model DE2.1; Delsys, Boston, MA) applied to lightly abraded skin over the respective muscle belly. Electrode placement for the ES muscle was $2 \mathrm{~cm}$ lateral to the spinous process; for Sol, about $2 \mathrm{~cm}$ distal to the medial head of the gastrocnemius (also see Winter 1991). Electrode placement was carefully chosen to minimize cross talk from adjacent muscles during isometric 
contractions (Ivanenko et al. 2006). Before the electrodes were placed, the subject was instructed about how to selectively activate each muscle (Kendall et al. 1993), while EMG signals were monitored. The signals were amplified $(\times 10,000)$, filtered $(20-450 \mathrm{~Hz}$; Bagnoli 16 , Delsys), and sampled at 1,000 Hz. Sampling of kinematic, force platform, and EMG data were synchronized.

At the end of the recording session, anthropometric measurements were taken on each subject. These included the mass and stature of the subject and the length and circumference of the main segments of the body (Zatsiorsky et al. 1990).

\section{Data analysis}

The body was modeled as an interconnected chain of rigid segments: GH-IL for the trunk, GH-Elb for the arm, Elb-Wri for the forearm, IL-GT for the pelvis, GT-LE for the thigh, LE-LM for the shank, and LM-VM for the foot. The elevation angle of each segment in the sagittal plane corresponds to the angle between the projected segment and the vertical. The main limb axis was defined as GT-LM. The elevation angle of each segment (including the limb axis) corresponds to the angle between the segment projected on the sagittal plane and the vertical (positive in the forward direction, i.e., when the distal marker falls anterior to the proximal one). Gait cycle was defined as the time between two successive foot-floor contacts by the same leg according to the local minima of the vertical displacement of the HE marker (Ivanenko et al. 2007). The timing of the lift-off was determined analogously (when the VM marker was elevated by $3 \mathrm{~cm}$ ).

For the analysis of locomotor patterns, the steps related to gait initiation and termination were discarded and only those performed in the central section of the path at about constant speed were included in the analysis. Subjects usually performed several steps with approximately a steady-state velocity (Fig. 1C). Walking speed for each stride was computed as the mean velocity of the horizontal trunk movement, the latter being identified by the time course of the displacement of a virtual marker located at the midpoint between left and right IL markers. Arm oscillations were estimated in both the sagittal and the frontal planes (as the peak-to-peak amplitude of the GH-Wri elevation angle). Head and shoulder yaw and roll rotations in space were computed, as well as the head and trunk pitch angles. For yaw and roll angle computation, two anterior head markers and two shoulder markers were used. For the trunk pitch angle, the long axis of the trunk was defined by connecting the midpoint of the two (left and right) IL markers with the midpoint of the two GH markers. For the head pitch angle, we used the anterior-posterior axis of the head defined by connecting the anterior head marker with the midpoint of the two anterior markers. The data for the left and right legs were pooled together for the analysis of gait kinematics. Data were time-interpolated over individual gait cycles to fit a normalized 200-point time base.

The time-varying friction at the foot-ground interface was calculated by dividing the instantaneous horizontal forces $\left(\mathrm{F}_{\mathrm{x}}^{2}+\mathrm{F}_{\mathrm{z}}^{2}\right)^{1 / 2}$ by the instantaneous vertical force $\mathrm{F}_{\mathrm{y}}$ (Fig. 2A). The average friction (Heiden et al. 2006) in mid- and late stances, where the foot slipped in the lateral direction, was also determined to characterize the operational coefficient of friction between the foot and the slippery surface during walking (see RESULTS).

The EMG records were numerically rectified, low-pass filtered using a zero-lag fourth-order Butterworth filter with a cutoff of $10 \mathrm{~Hz}$,
A

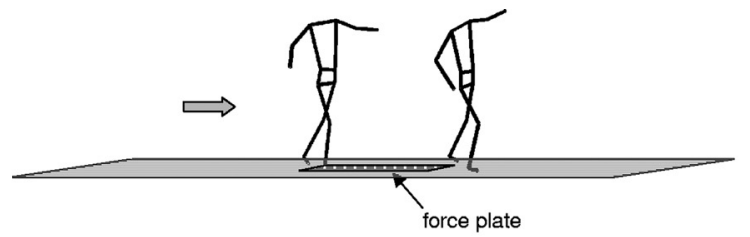

B
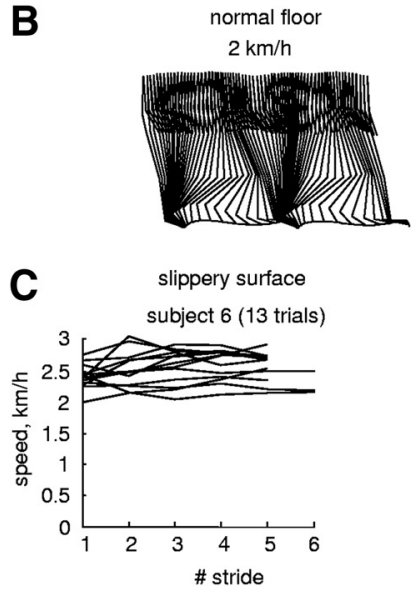

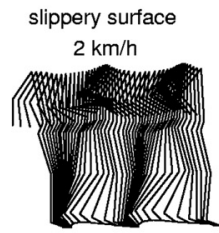

D slippery surface

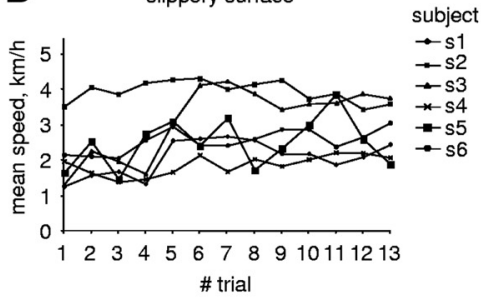

$\mathbf{E}$
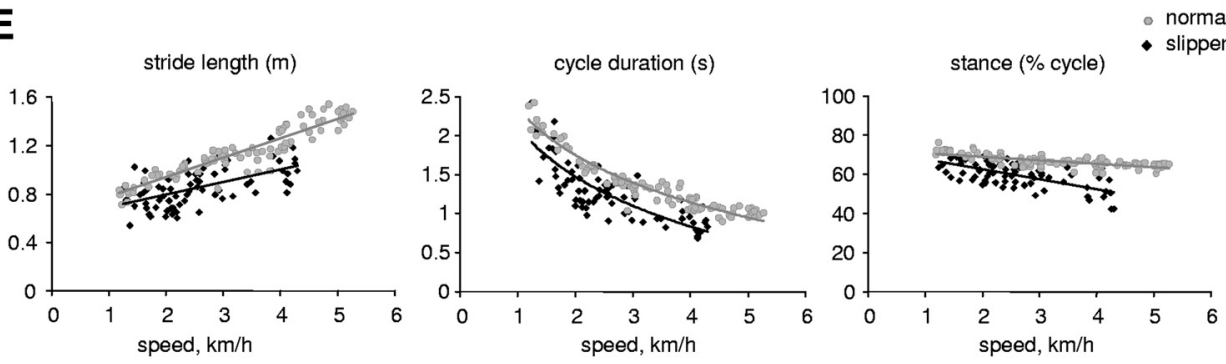

FIG. 1. General gait characteristics during walking on a normal floor and a slippery surface. A: schematic picture of the slippery walkway with a force plate and 2 bilateral stick diagrams of the subject. Note a wide lateral position of upper limbs while walking on the slippery surface. $B$ : unilateral stick diagrams of the 2 consecutive steps in one representative subject during walking at about $2 \mathrm{~km} / \mathrm{h}$. $C$ : speed changes during walking along a 7-m slippery walkway as a function of stride number in one subject across 13 trials. Note a relatively constant walking speed across consecutive steps in most trials. $D$ : mean walking speed as a function of trial number in 6 subjects. $E$ : comparison of stride length, cycle, and stance durations between slippery (black) and nonslippery (gray) surface as a function of walking speeds. Each point represents a single trial for all trials and subjects. The data were fitted by linear (left and right panels) and 2nd-order polynomial (middle panel) functions. 


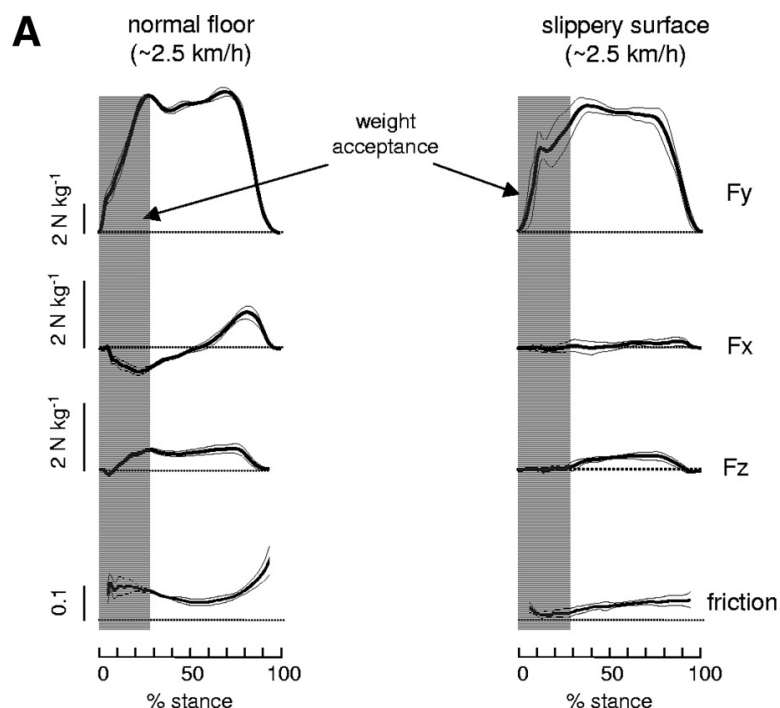

B

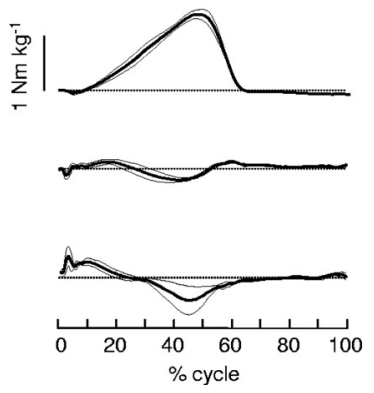

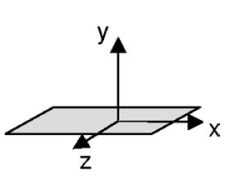

FIG. 2. Ground reaction forces and joint moments of force during walking on the slippery and nonslippery floor surface at matched walking speeds $(\sim 2.5 \mathrm{~km} / \mathrm{h})$ in one representative subject. $A$ : ensemble-averaged ( $\pm \mathrm{SD}, n=10$ steps) vertical, lateral, and anterior-posterior ground reaction forces and the time-varying utilized friction. The time-varying utilized friction was calculated by dividing the instantaneous horizontal forces $\left(\mathrm{F}_{\mathrm{x}}^{2}+\mathrm{F}_{\mathrm{z}}^{2}\right)^{1 / 2}$ by the instantaneous vertical force $\mathrm{F}_{\mathrm{y}}$. The patterns are computed and plotted vs. normalized stance. Note drastically decreased lateral and anterior-posterior shear forces and a typical prominent initial vertical loading peak during the weight-acceptance period (marked by a shaded area) on the slippery surface. $B$ : profiles of joint moments (normalized to body weight) throughout gait cycle. time-interpolated over individual gait cycles to fit a normalized 200-point time base, and averaged across steps and trials to obtain the ensemble-averaged EMG patterns.

\section{Calculation of the center-of-body mass (COM)}

The center of body mass position derived as

$$
C O M=\frac{\sum_{i}^{n} m_{i} \cdot \vec{r}_{i}}{\sum_{i}^{n} m_{i}}
$$

where $m_{i}$ and $r_{i}$ are the mass and position of the center of mass of each body segment, respectively, derived using measured kinematics, anthropometric data taken on each subject (see earlier text), and regression equations proposed by Zatsiorsky et al. (1990) for adults.

\section{Intersegmental coordination}

The intersegmental coordination in the sagittal plane was evaluated in position space as previously described (Bianchi et al. 1998; Borghese et al. 1996). The temporal changes of the elevation angles at the thigh, shank, and foot covary during walking. When these angles are plotted one versus the others in a three-dimensional graph, they describe a path that can be fitted (in the least-square sense) by a plane over each gait cycle. To this end, we computed the covariance matrix of the ensemble of time-varying elevation angles (after subtraction of their mean value) over each gait cycle. The three eigenvectors $u_{1}-u_{3}$, rank ordered on the basis of the corresponding eigenvalues, correspond to the orthogonal directions of maximum variance in the sample scatter. The first two eigenvectors $\mathrm{u}_{1}$ and $\mathrm{u}_{2}$ lie on the best-fitting plane of angular covariation. The third eigenvector $\left(u_{3}\right)$ is the normal to the plane and defines the plane orientation. For each eigenvector, the parameters $\mathrm{u}_{\mathrm{it}}, \mathrm{u}_{\mathrm{is}}$, and $\mathrm{u}_{\mathrm{if}}$ correspond to the direction cosines with the positive semiaxis of the thigh, shank, and foot angular coordinates, respectively. The orientation of the covariation plane in each condition was compared with the mean orientation of the corresponding plane in the normal floor walking condition at matched walking speeds. The planarity of the trajectories was quantified by the percentage of total variation (PV) accounted for by the first two eigenvectors of the data covariance matrix (for ideal planarity $\mathrm{PV}=100 \%$ and the third eigenvalue $=0$ ).

\section{Ground reaction forces and joint moments of force}

Only steps when one leg stepped onto the force plate were analyzed since they were not contaminated by the ground reaction forces produced by another leg. In general, from 3 to 10 such steps (depending on the subject) could be identified and analyzed across 13 recorded trials during walking on the slippery surface in each subject. The moments of forces (with extensor moments being positive) at the ankle, knee, and hip joints of the leg were calculated using measured kinematics, force plate data, anthropometric data taken on each subject, and the traditional Newton-Euler inverse dynamics model (Bresler and Frankel 1950). The forces and moments were normalized to the body mass (Winter 1991) and averaged across trials.

\section{Spatiotemporal patterns of MN activity in the spinal cord}

Because the method has been thoroughly documented in our previous reports (Ivanenko et al. 2006, 2008), here we describe it only briefly. The recorded patterns of EMG activity were mapped onto the 
approximate rostrocaudal location of ipsilateral $\mathrm{MN}$ pools in the human spinal cord. This approach provides information about pattern generator output in terms of segmental control rather than in terms of individual muscle control. In particular, it can be used to characterize network architecture for different gaits by considering relative intensities, spatial extent, and temporal structure of the spinal motor output (Cuellar et al. 2009; Golubitsky et al. 1999; Grasso et al. 2004; Ivanenko et al. 2008; Mentel et al. 2008; Yakovenko et al. 2002).

Briefly, the method was the following. In this study we used myotomal charts of Kendall et al. (1993). Despite likely anatomical variability (Phillips and Park 1991; Stewart 1992), the data from these charts appear sufficiently robust for the spatial resolution currently available in our reconstruction technique. Kendall et al. (1993) compiled reference segmental charts for all body muscles by combining the anatomical and clinical data from six different sources. A capital $\mathrm{X}$ in Kendall's chart denotes localization agreed on by five or more sources, a small $\mathrm{x}$ denotes agreement of three to four sources, and a bracketed (x) denotes agreement of only two sources. In our maps, $\mathrm{X}$ and $\mathrm{x}$ were weighted 1 and 0.5 , respectively, whereas we discarded (x). We assumed that our population of subjects has the same spinal topography as that of this reference population.

To reconstruct the output pattern of any given spinal segment $\mathrm{Sj}$ of the most active lumbosacral segments (L2-S2), all rectified EMG waveforms corresponding to that segment were averaged. The assumption implicit in this method is that the rectified EMG provides an indirect measure of the net firing of MNs of that muscle in the spinal cord (Day and Hulliger 2001; Hoffer et al. 1987). We used the nonnormalized procedure (EMGs were expressed in microvolts $[\mu \mathrm{V}]$ ) (Ivanenko et al. 2008)

$$
S_{j}=\frac{\sum_{i=1}^{n_{j}} k_{i j} \cdot E M G_{i}}{n_{j}}
$$

where $n_{j}$ is the number of $\mathrm{EMG}_{\mathrm{i}}$ waveforms corresponding to the $\mathrm{jth}$ segment and $\mathrm{k}_{\mathrm{ij}}$ is the weighting coefficient for the ith muscle (X and $\mathrm{x}$ in Kendall's chart were weighted with $\mathrm{k}_{\mathrm{ij}}=1$ and $\mathrm{k}_{\mathrm{ij}}=0.5$, respectively).

Using the Kendall chart results in the six rostrocaudal discrete activation waveforms since the anatomical data are broken up into the six vertebrate segments (L2-S2). To visualize a continuous smoothed rostrocaudal spatiotemporal activation of the spinal cord we used a filled contour plot that computes isolines calculated from the activation waveform matrix (6 segments $\times 200$ points) and fills the areas between the isolines using constant colors (the "contourf.m" function in Matlab). The Kendall chart indicates only the segments innervating each muscle and not the fraction of total motor pool of the muscle that can be assigned to a segment. However, we have previously shown (Ivanenko et al. 2006) that using the Sharrard (1964) data table for innervation, that approximates the proportion of total muscle activation attributable to each segment (by taking multiple slices within each spinal segment), instead of assuming equal proportions in all segments, gives similar loci of MN activity in the lumbosacral enlargement to those using the Kendall chart.

The temporal locus of MN activity in the lumbosacral spinal cord was estimated using a method similar to that of Yakovenko et al. (2002). We calculated the center of activity (CoA) of the six (from L2 to S2) most active lumbosacral segments using the following formula

$$
\operatorname{CoA}=\frac{\sum_{j=1}^{N} S_{j} \times \vec{j}}{\sum_{j=1}^{N} S_{j}}
$$

where $S_{\mathrm{j}}$ is the estimated activity (from $E q$. 2) of the jth segment (the origin for the $\mathrm{CoA}$ and for the vector $\mathrm{j}$ being defined as the caudalmost segment) and $\mathrm{N}$ is the number of segments ( $n=6$ for the Kendall charts). Thus the center of activity was expressed in terms of absolute position within the lumbosacral enlargement. At any time there was typically only one dominant locus of activity in the lumbosacral enlargement during normal walking (Ivanenko et al. 2006), although it may not be the case for walking on the slippery surface (see RESULTS). Therefore the CoA can be considered only as a qualitative parameter since averaging between distinct foci of activity (for instance, L2 and S2) may lead to misleading activity in the midsegments ( $\sim$ L5).

The mean activation of the lumbosacral enlargement in the gait cycle was also calculated and compared across conditions (slippery vs. nonslippery surface) at matched walking speeds.

\section{Statistics}

The kinematics and EMG patterns of walking on the slippery surface (protocol 2) were assessed by comparing locomotor patterns with matching speeds on the nonslippery surface (protocol 1) for each subject. Descriptive statistics included means \pm SD of the mean. Student's $t$-tests were used to compare gait parameters between normal and slippery-surface conditions. Reported results are considered significant for $P<0.05$. Statistics on correlation coefficients was performed on the normally distributed, Z-transformed values. Crosscorrelation functions between pairs of EMGs (after subtraction of the respective means) were computed as previously described (Grasso et al. 1998, 2000). The data analysis and spinal MN activity map construction were performed with software written in Matlab (R2008, The MathWorks).

\section{RES U L T S}

\section{General characteristics of walking on the slippery surface}

Subjects adopted a significantly slower speed than normal when walking on a slippery surface $(2.8 \pm 0.5$ vs. $4.8 \pm 0.9$ $\mathrm{km} / \mathrm{h}$ on a normal surface, $P<0.001$, paired $t$-test) (see also Fong et al. 2008b). Even when speeds were matched, their stride length was significantly shorter on the slippery than that on the normal surface (Fig. 1E, left plot). As a result, cycle duration was also shorter and the relative stance duration (percentage of cycle) also tended to decrease (Fig. 1E, middle and right plots). With repeated trials, the subjects had a tendency to walk faster and to decrease the cycle duration. This is evident in the comparisons between the first and last trials in Table 1 .

The stick diagram in Fig. $1 B$ illustrates a typical template of the kinematics of stepping by the same subject at about $2 \mathrm{~km} / \mathrm{h}$ on the two surfaces. Note the shorter step and the smaller limb inclination angle at heel contact (more vertical limb orientation). Note also the larger excursion of upper limbs while walking on the slippery surface (Fig. 1, $A$ and $B$ ).

\section{Gait kinetics}

Figure $2 A$ illustrates ground reaction forces for one representative subject walking on the two surfaces at the same mean speed $(\sim 2.5 \mathrm{~km} / \mathrm{h})$. The ground reaction forces on the nonslippery floor were typical of those reported in the literature (Winter 1991). The normal forces $\left(\mathrm{F}_{\mathrm{y}}\right.$, perpendicular to the walking surface) reach a maximum during the initial stance phase and another one in the final phase of stance. The anterior-posterior shear forces $\left(\mathrm{F}_{\mathrm{x}}\right.$, along the direction of locomotion) exhibit a biphasic, symmetrical shape first in the forward direction and then in the rearward direction. The first peak in shear force is considered to be critical with respect to slips resulting in falls 
TABLE 1. Gait parameters, amplitudes (peak-to-peak) of movements, and mean lumbosacral MN activation in the first step of the first trial and the averaged parameters across all steps in the first and last trials ( $\pm S D, n=6)$ during walking on the slippery surface

\begin{tabular}{|c|c|c|c|}
\hline Variable & $\begin{array}{l}\text { First Step } \\
\text { First Trial }\end{array}$ & First Trial & Last Trial \\
\hline Speed, $\mathrm{km} / \mathrm{h}$ & $1.78 \pm 0.79$ & $1.96 \pm 0.83$ & $3.15 \pm 0.91 *$ \\
\hline Stride, $\mathrm{m}$ & $0.79 \pm 0.13$ & $0.83 \pm 0.11$ & $0.78 \pm 0.09$ \\
\hline Cycle, s & $1.76 \pm 0.54$ & $1.71 \pm 0.60$ & $1.17 \pm 0.19 *$ \\
\hline Stance, \% & $67.16 \pm 5.68$ & $70.40 \pm 8.05$ & $61.86 \pm 4.95^{*}$ \\
\hline Thigh, deg & $37.80 \pm 5.23$ & $36.30 \pm 7.68$ & $37.60 \pm 8.41$ \\
\hline Shank, deg & $48.20 \pm 5.02$ & $47.50 \pm 5.23$ & $51.70 \pm 9.11$ \\
\hline Foot, deg & $46.60 \pm 5.85$ & $46.50 \pm 5.43$ & $52.80 \pm 10.70$ \\
\hline Gait loop area, $\operatorname{deg}^{2}$ & $1,494 \pm 191$ & $1,126 \pm 320$ & $1,434 \pm 403$ \\
\hline $\mathrm{VM}_{\mathrm{y}}, \mathrm{cm}$ & $7.51 \pm 1.79$ & $8.72 \pm 2.71$ & $8.38 \pm 2.09$ \\
\hline $\mathrm{VM}_{\mathrm{z}}, \mathrm{cm}$ & $10.30 \pm 2.73$ & $11.62 \pm 2.59$ & $10.04 \pm 2.33$ \\
\hline Foot yaw, deg & $24.10 \pm 12.01$ & $26.47 \pm 15.90$ & $33.72 \pm 14.78$ \\
\hline Head yaw, deg & $5.45 \pm 1.80$ & $5.95 \pm 1.35$ & $6.21 \pm 1.67$ \\
\hline Shoulder yaw, deg & $19.60 \pm 2.05$ & $18.08 \pm 5.81$ & $17.96 \pm 6.71$ \\
\hline Arm sagittal, deg & $41.40 \pm 14.41$ & $41.57 \pm 14.39$ & $47.37 \pm 23.57$ \\
\hline Arm frontal, deg & $24.40 \pm 3.67$ & $22.21 \pm 7.25$ & $26.28 \pm 8.55$ \\
\hline $\mathrm{GT}_{\mathrm{z}}, \mathrm{cm}$ & $6.74 \pm 2.39$ & $6.46 \pm 2.01$ & $2.06 \pm 0.35 * * *$ \\
\hline $\mathrm{GT}_{\mathrm{y}}, \mathrm{cm}$ & $4.17 \pm 0.57$ & $4.28 \pm 0.85$ & $3.04 \pm 0.56$ \\
\hline $\mathrm{GH}_{\mathrm{z}}, \mathrm{cm}$ & $12.60 \pm 1.08$ & $13.13 \pm 2.62$ & $11.66 \pm 2.21$ \\
\hline $\mathrm{GH}_{\mathrm{y}}, \mathrm{cm}$ & $7.45 \pm 2.06$ & $6.01 \pm 0.85$ & $5.30 \pm 0.90$ \\
\hline $\mathrm{GH}_{\mathrm{z}}-\mathrm{GT}_{\mathrm{z}}, \mathrm{cm}$ & $12.19 \pm 2.15$ & $13.31 \pm 1.65$ & $10.98 \pm 2.06$ \\
\hline $\begin{array}{l}\text { Mean lumbosacral MN } \\
\text { activation, } \mu \mathrm{V}\end{array}$ & $14.00 \pm 6.00$ & $14.00 \pm 5.00$ & $18.00 \pm 6.00$ \\
\hline
\end{tabular}

Asterisks denote significant differences: $* P<0.05$; $* * * P<0.001$.

(Redfern et al. 2001). The transverse shear force $\left(\mathrm{F}_{\mathrm{z}}\right)$ has a shape similar to that of $\mathrm{F}_{\mathrm{y}}$ (Fig. 2A, left).

On the slippery surface, the normal forces demonstrated the typical peak at the onset of stance likely due to a rigid vertical foot impact (with a nonzero vertical foot velocity). However, the horizontal shear forces were very small during the weight-
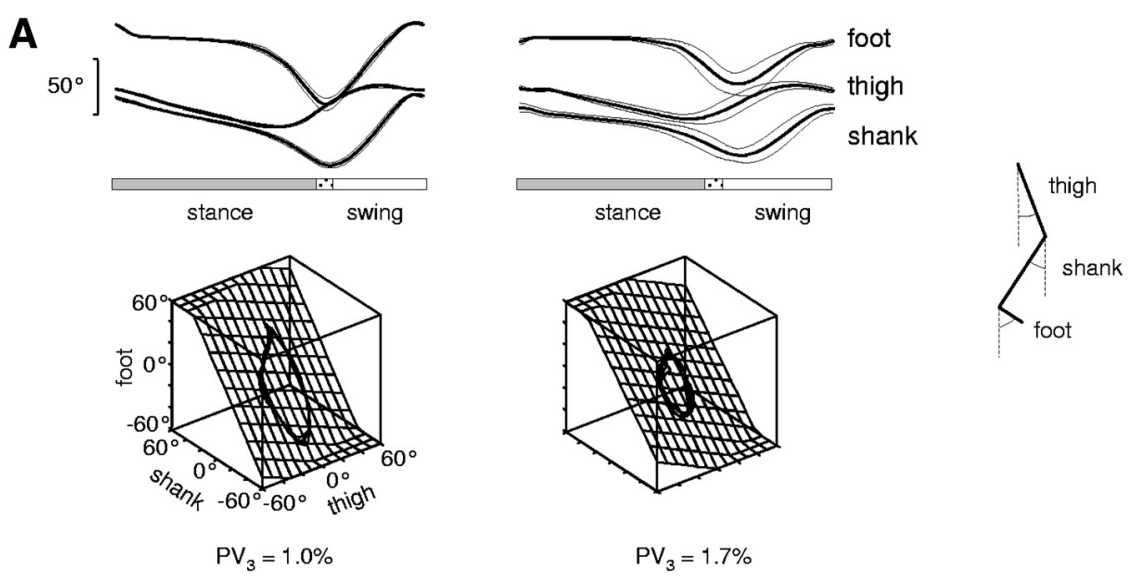

B

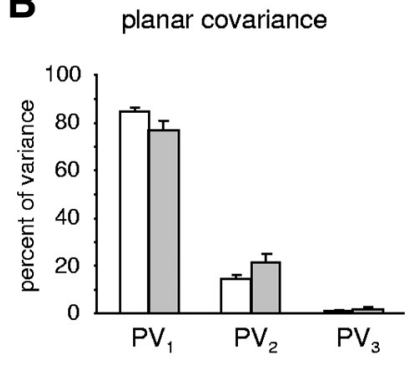

covariance plane orientation

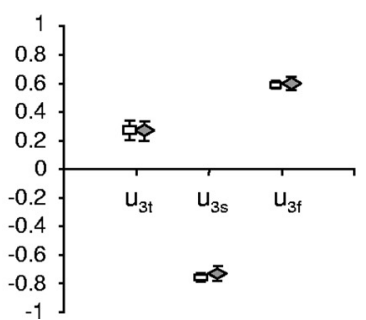

acceptance phase (first 20\% of stance; see shaded area in Fig. $2 A$ ) and did not show a systematic behavior in all participants. The mean anterior-posterior shear force during the weightacceptance phase on the slippery surface was $0.02 \pm 0.06 \mathrm{~N} / \mathrm{kg}$ and did not differ significantly from zero $(P=0.27$, one-tailed $t$-test). The lateral shear force was $0.05 \pm 0.05 \mathrm{~N} / \mathrm{kg}$ and differed from zero $(P=0.16$, one-tailed $t$-test). However, both anterior-posterior and lateral horizontal forces $(-0.46 \pm 0.11$ and $0.28 \pm 0.07 \mathrm{~N} / \mathrm{kg}$, respectively) during the weight-acceptance phase were significantly larger on the nonslippery surface $(P<0.0003$, paired $t$-test $)$. The anterior-posterior shear forces $\left(\mathrm{F}_{\mathrm{x}}\right)$ remained small throughout the whole stance phase, whereas the transverse forces $\left(\mathrm{F}_{\mathrm{z}}\right)$ were directed laterally during mid- and late stances (Fig. 2).

The time-varying friction, calculated by dividing the instantaneous resultant horizontal force $\left(\mathrm{F}_{\mathrm{x}}^{2}+\mathrm{F}_{\mathrm{z}}^{2}\right)^{1 / 2}$ by the instantaneous vertical force $F_{y}$, was lower on the slippery surface, especially during the weight-acceptance phase (Fig. $2 A$, bottom). The average friction in late stance (between 60 and $90 \%$ of stance) was $0.06 \pm 0.01$ across all subjects and characterized the operational coefficient of friction during walking on the slippery surface, when there were slight lateral slips (see following text, Fig. 4). In contrast, the average friction in the late stance during normal walking was $0.09 \pm 0.02$ at the same mean walking speed (although the foot did not slip and thus the friction force did not reach its maximal value).

Moments of force at the ankle, knee, and hip joints also showed specific features on the slippery surface. Characteristic profiles are shown in Fig. $2 B$. Notice the greater intertrial variability of both contact forces and joint moments of force on the slippery surface. The moment at the ankle joint tended to demonstrate a biphasic behavior with a noticeable initial peak, although the maximal amplitude of the ankle joint moment was smaller on the slippery surface $(P<0.01$, paired $t$-test $)$. During

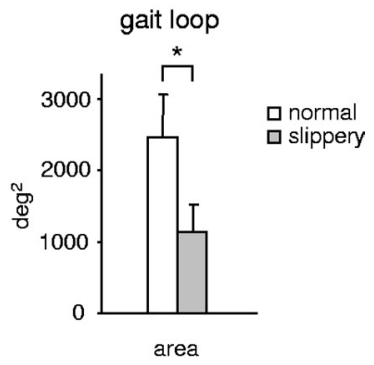

FIG. 3. Intersegmental coordination during walking on normal and slippery surfaces. $A$ : thigh, shank, and foot elevation angles $( \pm \mathrm{SD})$ in the sagittal plane averaged across all trials and subjects, and corresponding gait loops and interpolation planes at matched walking speeds. Three-dimensional gait loops represent one gait cycle obtained by plotting the thigh waveform vs. the shank and foot waveforms (after subtraction of mean values). The kinematic waveforms are plotted vs. normalized gait cycle. Because the relative duration of stance varied across subjects, a hatched region indicates an amount of variability in the stance phase duration across subjects. The interpolation planes result from orthogonal planar regression. Gait cycle paths progress in time in the counterclockwise direction, heel touch-down and toe-off phases corresponding roughly to the top and bottom of the loops, respectively. $B$ : percentage of total variation (PV) explained by each principal component (left), the orientation of the plane (normal to the plane: $\mathrm{u}_{3 \mathrm{t}}$, $\mathrm{u}_{3 \mathrm{~s}}$, and $\mathrm{u}_{3 \mathrm{f}}$ projections; middle), and the area of the gait loop (right) are indicated for each gait. Note similar plane orientation on the slippery surface but significantly smaller gait loop. 
weight acceptance (first $20 \%$ of stance), the maximally achieved absolute value of all moments was significantly higher than that during normal walking $(P<0.05$ for all three moments, paired $t$-test).

Therefore several features of gait kinetics during normal walking and walking on the slippery surface are different. The main distinction of the slippery gait is the near absence of tangential shear forces during the weight-acceptance part of the stance phase.

\section{Gait kinematics}

During normal walking, the temporal changes in the elevation angles of lower limb segments (thigh, shank, and foot) covary on a plane, describing a characteristic loop over each stride (Fig. 3A, left) (Borghese et al. 1996). The gait loop and its associated plane depend on the amplitude and phase of the limb segment oscillations (Barliya et al. 2009; Courtine and Schieppati 2004; Lacquaniti et al. 1999, 2002) and reflect foot motion in different gaits (Ivanenko et al. 2007). Although the angular motion was more variable across steps under lowfriction conditions, the trajectories of the limb segment angles were still close to a plane (Fig. 3A). This is evidenced by the small contribution made by the third eigenvector $\left(\mathrm{PV}_{3}\right)$ to the total angular variance. The segment angle trajectory variance accounted for by $\mathrm{PV}_{3}$ on the normal surface was $0.8 \pm 0.2 \%$ and $1.7 \pm 0.8 \%$ on the slippery surface (Fig. $3 B$, left).

The orientation of the covariation plane (the normal to the plane) was not significantly different in the two walking conditions (Fig. 3B, middle). However, the shape of the loop was less elongated and it was significantly smaller during walking on the slippery surface (the area of the loop was about half, $P<0.001$, paired $t$-test, Fig. $3 B$, right), reflecting changes in the angular amplitudes and in the foot kinematics.

Foot motion on the slippery surface resembled that of a nonplantigrade gait (without heel-to-toe rolling pattern) and was characterized by a larger foot clearance and a single-peak foot trajectory (Fig. 4A, top plots). Lateral foot movements were significantly enhanced $(P<0.01$, paired $t$-test, Fig. $4 B)$ due to slips in the mid- and late stances (by a few centimeters; Fig. $4 A$, middle traces) but with less outward-pointing of the toes throughout the gait cycle (Fig. 4A, bottom traces).

Consistent and systematic trunk tilts in the frontal plane were idiosyncratic features of walking on the slippery surface (Fig. 5, $A$ and $D$ ). This is in contrast to normal walking, where both the hip and shoulder moved in concert, resulting in parallel lateral trunk shifts without noticeable inclinations. On the slippery surface, lateral hip $\left(\mathrm{GT}_{\mathrm{z}}\right)$ displacements decreased, whereas shoulder $\left(\mathrm{GH}_{\mathrm{z}}\right)$ oscillations increased in all subjects, resulting in the prominent trunk tilts in the frontal plane. Note also a "guard arms" position and the lateral arm motion during walking on the slippery surface (Fig. 5, $A$ and $C$ ). Vertical motion of the trunk (Fig. 5B) and COM (Fig. 5E) was still present during walking under the low-friction conditions, although lateral hip and COM displacements were significantly reduced (Fig. 5, $D$ and $E, P=0.004$ ).

As a consequence of the lateral trunk inclination on slippery surfaces, the head also exhibited alternating lateral tilts (roll) (Fig. 6A, bottom). However, the amplitude of these oscillations was less than that of the trunk $(P<0.05$, paired $t$-test $)$, suggesting a head-stabilization strategy (Hirasaki et al. 1999;

A

foot motion

normal floor
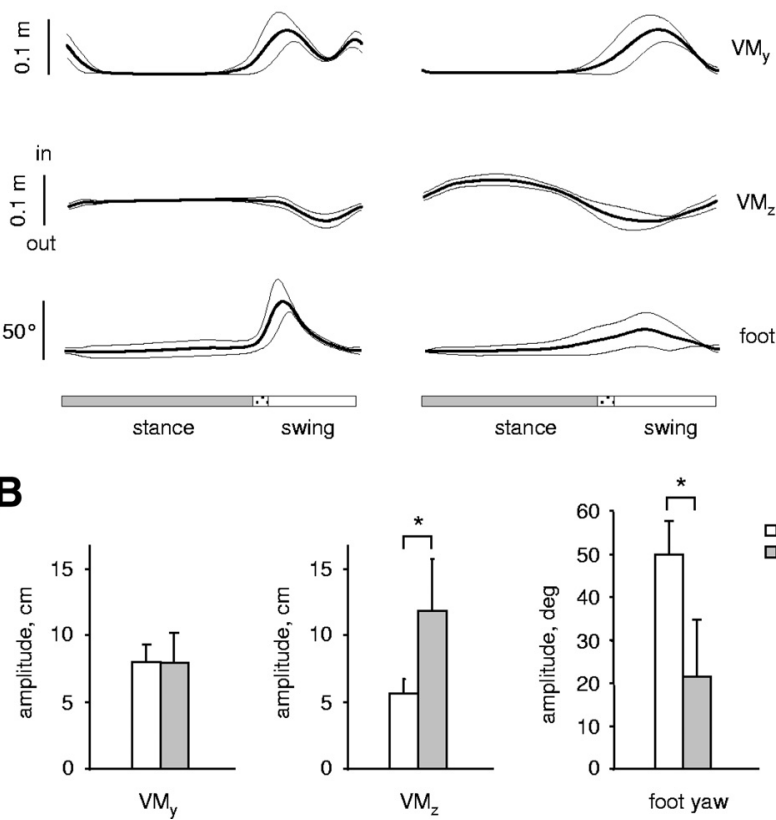

FIG. 4. Characteristics of foot motion. $A$ : ensemble-averaged ( $\pm \mathrm{SD}, n=6$ ) vertical and lateral foot (VM marker) movements and the horizontal yaw angle changes averaged across all trials and subjects during walking on the normal and slippery surfaces at matched walking speeds. The patterns are plotted vs. normalized gait cycle, upward direction corresponding to the outward veering of the yaw foot angle. Note systematic nonstationary foot behavior (slipping by a few centimeters in the lateral direction) on the slippery surface during the stance phase. $B$ : peak-to-peak amplitudes. Asterisks denote significant differences across walking conditions $(P<0.05$, paired $t$-test $)$.

Menz et al. 2003; Pozzo et al. 1990). A similar stabilization was observed for the yaw rotations: the shoulders oscillated more on the slippery surface than on the normal floor, whereas the head yaw angle tended to decrease $(P=0.06$, Fig. 6$)$. The head and trunk pitch angle oscillations were small (a few degrees) and comparable on the slippery and normal floor.

Thus there are several features of the gait kinematics during slippery walking that are clearly different from normal walking. The most striking kinematics features of the slippery walking are the nonplantigrade gait and the single-phase trajectory of the foot during swing.

\section{Adaptive attenuation of lateral hip and COM movements over repeated trials}

To check whether there was any adaptation or other effect of practice on the slippery surface, we compared gait kinematics across repeated trials $(n=13$ total). In general, the kinematic variables were more variable in the first trial; however, the basic features of walking on the slippery surface were already present. In particular, the results revealed similar segment elevation angular amplitudes, vertical and lateral foot movements, and trunk tilts in the frontal plane between the first and last trials $(P>$ 0.05 for all the above-cited variables, Table 1). However, lateral hip and COM displacements in the first trial were comparable to those on the nonslippery surface $(P>0.6)$ but decreased significantly $(P<0.005)$ with trial repetition (Fig. 7). 
A
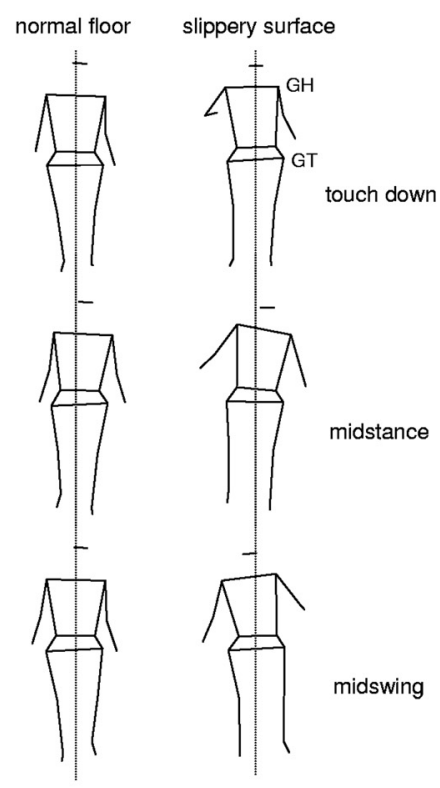

B
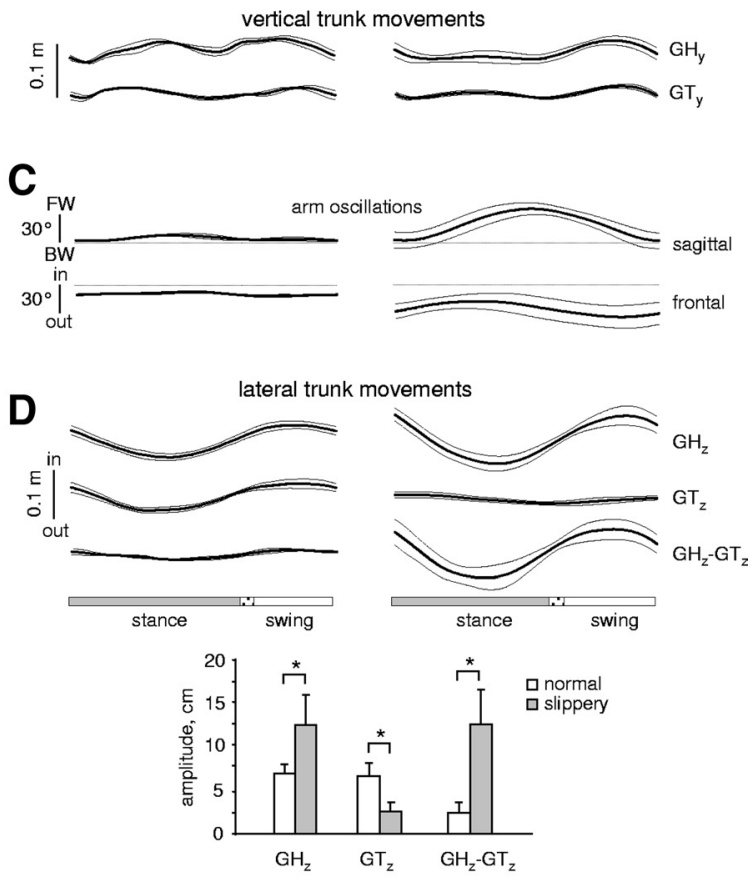

E
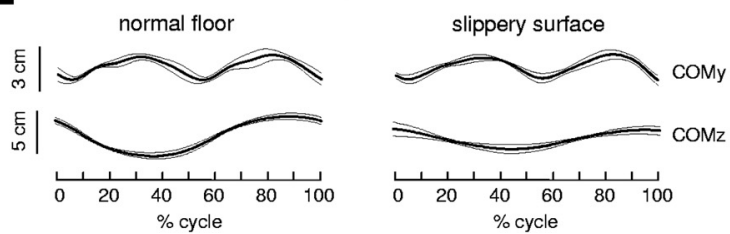

EMG patterns

We recorded EMG activity from 16 ipsilateral muscles on each side of the body. An example of muscle activation patterns is illustrated in Fig. 8. The amplitude of muscle activity was systematically higher on the slippery surface during both stance and swing and the relative burst duration tended to be longer. Furthermore, some muscles, which are basically silent in most subjects during normal walking at speeds $<3 \mathrm{~km} / \mathrm{h}$ (i.e., RF, VL, VM, SART; Ivanenko et al. 2006; Pepin et al. 2003), were always active in all subjects on the slippery surface.

In addition to the activation amplitude, the pattern and sequence of activation of individual muscles were substantially different on the slippery surface. Notice, for instance, the differences in midstance activity in the TFL, SART, Gmed, BF, VL, VM, and RF for the slippery surface (Fig. 9A). This was also shown by considering the cross-correlation functions of pairs of EMG ensemble averages of leg extensor muscles (Fig. 10). In particular we observed considerable shifts in the maximum of several cross-correlation functions (e.g., between thigh and calf extensor muscles, Fig. 10). For instance, a positive cross-correlation at a positive delay indicates a time lag of the LG, MG, and SOL muscles relative to RF, VL, and VM muscles on the normal floor by about $35-40 \%$ of gait cycle. On the slippery surface, the maximum of cross-correlation functions could shift to a quasi-agonistic activity in the same pair of muscles (e.g., between VL and SOL in Fig. 10).

Furthermore, the activity of muscles within synergistic groups was partially uncoupled in response to altered mechan-
FIG. 5. Trunk and center-of-body mass (COM) movements. A: stick diagrams in the frontal plane during 3 selected phases of the gait cycle in one representative subject. Note a "guard arms" position and their lateral motion along with the lateral trunk tilts during walking on the slippery surface. $B$ : vertical motion of the right shoulder $\left(\mathrm{GH}_{\mathrm{y}}\right)$ and hip $\left(\mathrm{GT}_{\mathrm{y}}\right)$ markers averaged across all trials and subjects at matched walking speeds. $C$ : arm oscillations in the sagittal and frontal planes averaged across all trials and subjects. $D$ : ensemble-averaged lateral trunk movements (right $\mathrm{GH}_{\mathrm{z}}$ and $\mathrm{GT}_{\mathrm{z}}$ and their differences are shown). Note significantly smaller lateral hip $\left(\mathrm{GT}_{\mathrm{z}}\right)$ displacements but augmented shoulder $\left(\mathrm{GH}_{\mathrm{z}}\right)$ oscillations, resulting in the idiosyncratic lateral trunk inclinations $\left(\mathrm{GH}_{\mathrm{z}}-\mathrm{GT}_{\mathrm{z}}\right)$ on the slippery surface. $E$ : vertical and lateral COM movements averaged across all trials and subjects. Data were plotted vs. a normalized cycle. Note significantly decreased COM oscillations in the frontal plane.

correlation coefficient between LG and MG activity for the period of stance was $0.89 \pm 0.06$ during walking and $0.91 \pm$ 0.04 during running and between LG and SOL, $0.82 \pm 0.10$ and $0.92 \pm 0.03$, respectively, indicating highly synchronized activation of these agonist muscles. In contrast, on the slippery surface we often observed multiple bursts of EMG and decoupled activation of calf muscles (Fig. 11A, right; see also uncoupling of anatomical synergists in Courtine and Schieppati 2003; Wakeling and Horn 2009). The correlation coefficient between LG and MG was $0.57 \pm 0.19$ and between LG and SOL $0.35 \pm 0.18$ (averaged across all trials, steps, and subjects). Moreover, the timing of the total activation of all calf muscles, estimated as the timing of the main peak of the averaged MG, LG, SOL waveform, also differed across walking conditions. The posterior calf muscles are active at roughly $70 \%$ of stance during normal walking and about $40 \%$ of stance during running (Fig. 11, $B$ and $C$, left). With walking on the slippery surface, this pattern was distributed widely across the stance phase (from 20 to $100 \%$ of stance, Fig. 11, $B$ and $C$, right).

The features of the EMG patterns that distinguished normal walking from walking on a slippery surface, which included the coactivation of RF and VM with the LG-MG-SOL muscles (Fig. 
A
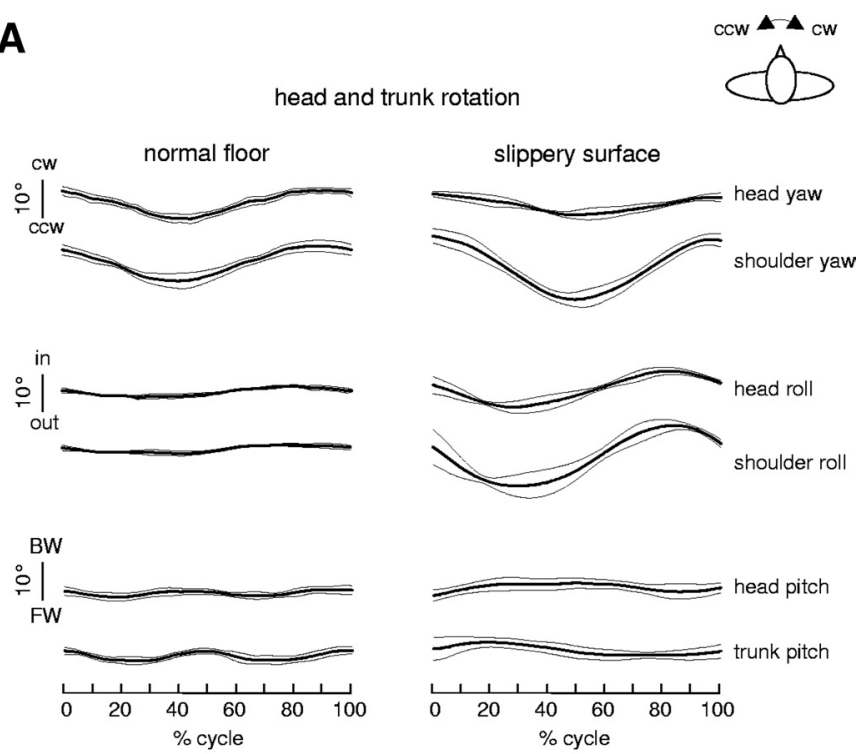

B
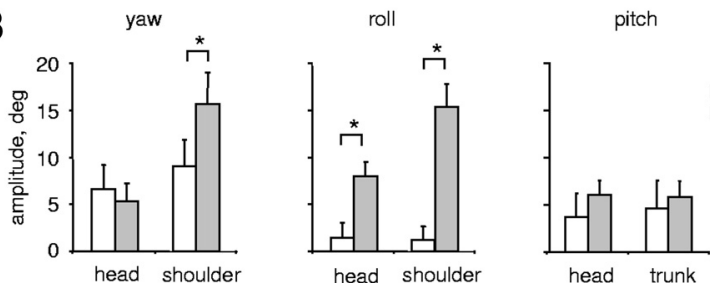

FIG. 6. Head and trunk rotations. A: yaw and roll rotation of the head and shoulders and pitch rotation of the head and trunk averaged across all trials and subjects. $B$ : peak-to-peak amplitudes. Asterisks denote significant differences across walking conditions $(P<0.05$, paired $t$-test $)$.

$9 A)$, were present with the first step on the slippery surface and persisted without significant change throughout all subsequent trials (Table 2). We found that the muscle-by-muscle waveforms of the EMG for the first step in the first trial were consistently correlated with the average waveform for each successive trial (average across trials $r=0.795$ ) and poorly correlated with the average EMG recorded during normal walking $(r=0.39$, Table 2 ). Moreover, the result was equivalent when the correlation was made with respect to the first step of the last trial.

\section{Spatiotemporal distribution of lumbosacral MN activity}

Despite some variability in the EMG patterns across muscles and steps, the overall organization of the MN activity showed systematic gait-dependent features (Fig. 9, $A$ and $B$ ). We used the EMG data to construct maps of spinal MN activity by adding up the contributions of each muscle to the total activity in each spinal segment (Ivanenko et al. 2006). The maps cover spinal levels L2-S2, corresponding to the levels of the MNs innervating the 16 recorded muscles. In general, each muscle is innervated by several spinal segments and each segment supplies several muscles (Kendall et al. 1993; Sharrard 1955, 1964). We focused on the lumbosacral enlargement because it contains the MNs of all the leg muscles.

The activation patterns tended to be segregated in spinal segments in a gait-specific manner. Figure $9 B$ illustrates the resulting averaged maps of MN activity during walking on the normal floor and slippery surface at matched walking speeds $(2.7 \pm 0.7$ and $2.8 \pm 0.6 \mathrm{~km} / \mathrm{h}$, respectively). The pattern is plotted versus normalized gait cycle. The EMG data show clear temporal patterns that are evident in the maps projected onto the spinal segments. As we reported previously (Ivanenko et al. 2006), for normal walking there are at least four major loci of activation in the lumbosacral enlargement corresponding to the four basic temporal components. A prominent fifth burst was also present in the thoracic and cervical spinal cord (not shown here). We also used the locus of the center of mass of MN activity (CoA) to describe how activity centers shifted in time through the step cycle (Fig. 9B, solid lines). In general, the CoA shows that rostral and caudal shifts occurred in two cycles for each step, the most prominent activity occurring during stance. In contrast, on the slippery surface we did not find any systematic CoA behavior and its rostral and caudal shifts were much smaller than those for normal walking. In addition, the loci of activation were also wider and stronger on the slippery surface (Fig. 9B).

As expected from the more intense EMG activity (Fig. 8), we found that the mean level of lumbosacral segment activity was about twofold greater on the slippery surface $(P<0.0001$, paired $t$-test, Fig. $9 C$ ) and it reflected increased activity in both proximal and distal leg muscles.

\section{I S C U S S I O N}

In this study we tested the hypothesis that slippery-surface walking takes place with a unique and specific gait rather than a continuous series of adaptations during otherwise normal walking. The evidence presented here supports the hypothesis by showing that the kinematics, kinetics, and muscle activation patterns are systematically different on the slippery and nonslippery surfaces, suggesting the existence of two distinct gait modes. Moreover the finding that the change in behavior can occur with the first step on the slippery surface provides evidence that the slippery gait is not simply an adaptation of normal walking to surface conditions (Tables 1 and 2).

However, some adaptation does take place. We also showed that lateral hip and COM oscillations were significantly reduced with continued experience on the slippery surface. Thus it seems that the subjects may have adopted a specific gait mode when they became aware of the unknown or abnormal surface conditions and then they adapted to the specific (slippery) condition they found (see also Cham and Redfern 2002; Heiden et al. 2006).

\section{Gait mode}

The gait parameters that were adopted by subjects when they expected to be walking on a slippery surface were characteristic across subjects and different from those of normal walking. They used a shorter stride length and stance duration even for speeds matched to those of normal walking (see also Cham and Redfern 2002; Lockhart et al. 2007; Fig. 1).

The kinetic patterns included a significant reduction in the anterior-posterior shear forces and correspondingly lower operational coefficient of friction (Fig. 2; Cham and Redfern 2002). The low friction surface means that the horizontal shear forces are significantly smaller (Fig. 2) (Heiden et al. 2006; Marigold and Patla 2002; Strandberg 1983). Nevertheless, the normal forces were more typical of walking on a nonslippery 
<smiles>CC1C2CC3CC1C2C3C</smiles>

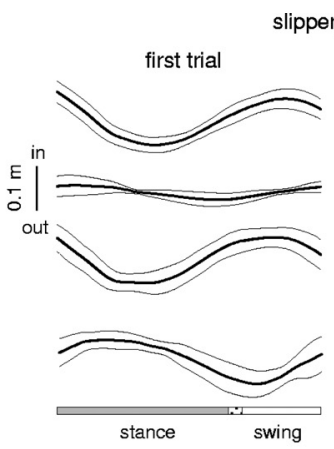

\section{B}

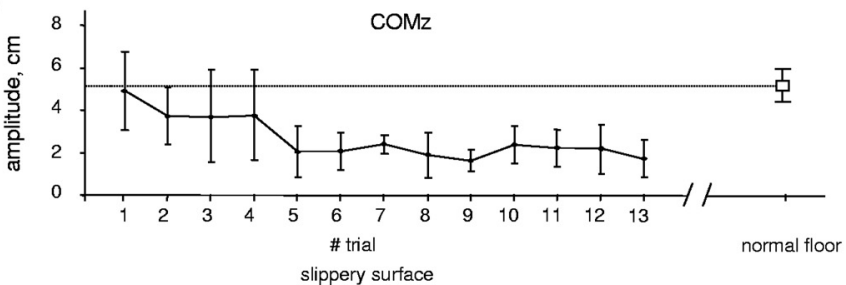

$\square$ first trial

last trial
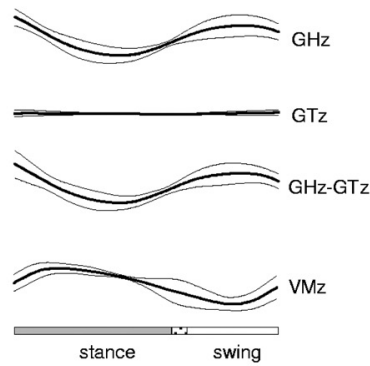

VM

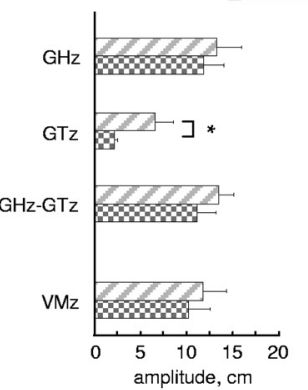

FIG. 7. Adaptive attenuation of lateral hip and COM movements on the slippery surface over repeated trials. $A$ : ensemble-averaged ( $\pm \mathrm{SD}, n=6)$ trunk $\left(\mathrm{GH}_{\mathrm{z}}, \mathrm{GT}_{\mathrm{z}}\right.$, and $\left.\mathrm{GH}_{\mathrm{z}}-\mathrm{GT}_{\mathrm{z}}\right)$ and foot $\left(\mathrm{VM}_{\mathrm{z}}\right)$ movements in the first and last trials (left) and corresponding peak-to-peak amplitudes (right). Note the presence of idiosyncratic lateral trunk inclinations $\left(\mathrm{GH}_{\mathrm{z}}-\mathrm{GT}_{\mathrm{z}}\right)$ already in the first trial and a significant decrement of lateral hip oscillations in the last trial. $B$ : changes in the amplitude of lateral COM movements with trial repetition. The value corresponding to walking on the nonslippery floor is indicated by the horizontal dotted line for comparison. surface, although they demonstrated an atypical peak at the onset of stance likely due to a rigid vertical foot impact, and the anterior-posterior shear forces remained small throughout the stance phase. Meanwhile, the lateral forces exhibited a system- atic lateral component during mid- and late stances (Fig. 2A). Characteristic moments at the ankle, knee, and hip joints also showed specific features that were different from those during normal walking (Fig. 2B).
A
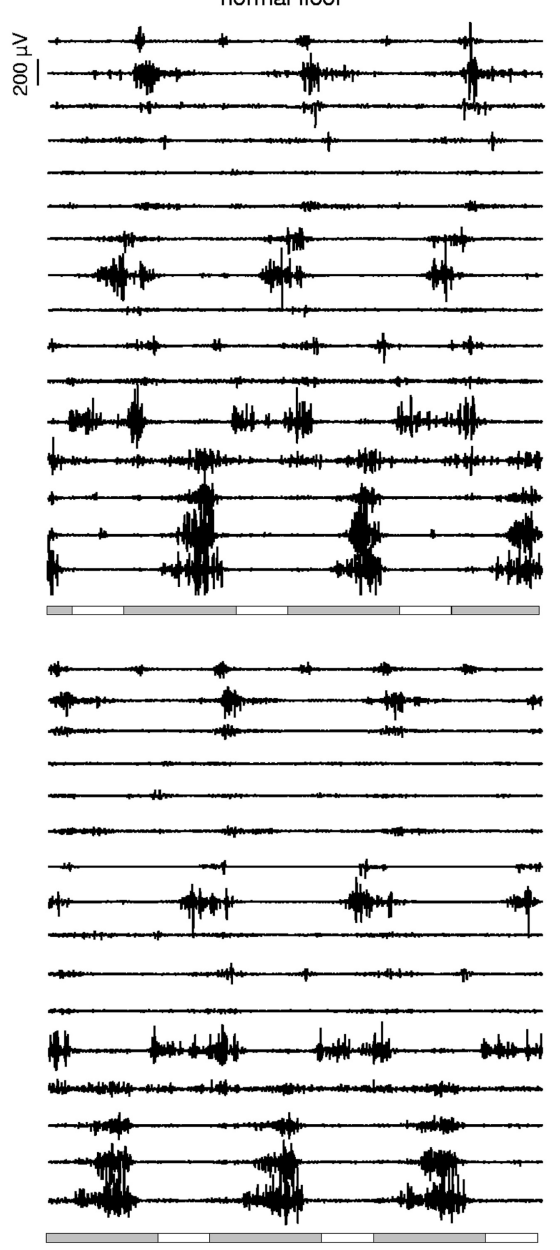

$1 \mathrm{~s}$
B
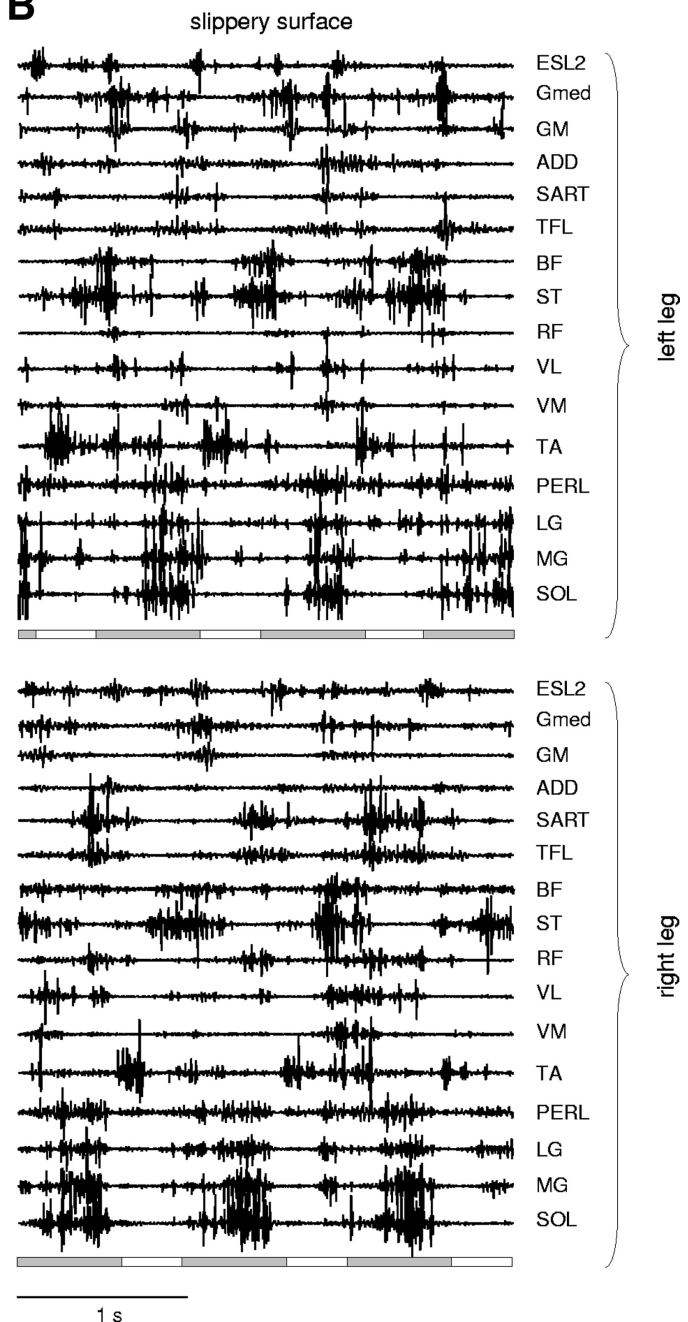

J Neurophysiol • VOL $103 \cdot$ FEBRUARY $2010 \cdot$ www.jn.org
FIG. 8. An example of bilateral electromyographic (EMG) activity in one representative subject during walking on the nonslippery and slippery surface at the same speed $(3.5 \mathrm{~km} / \mathrm{h})$. Three consecutive strides are shown in each case. 
A

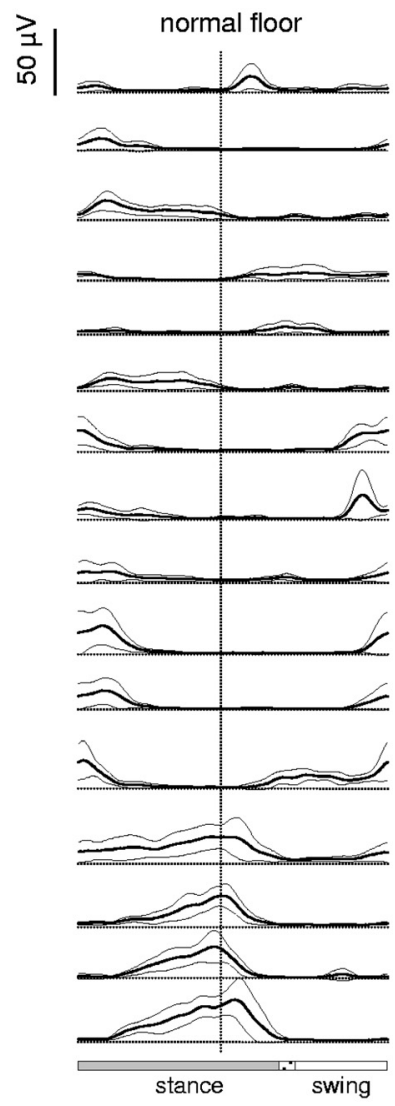

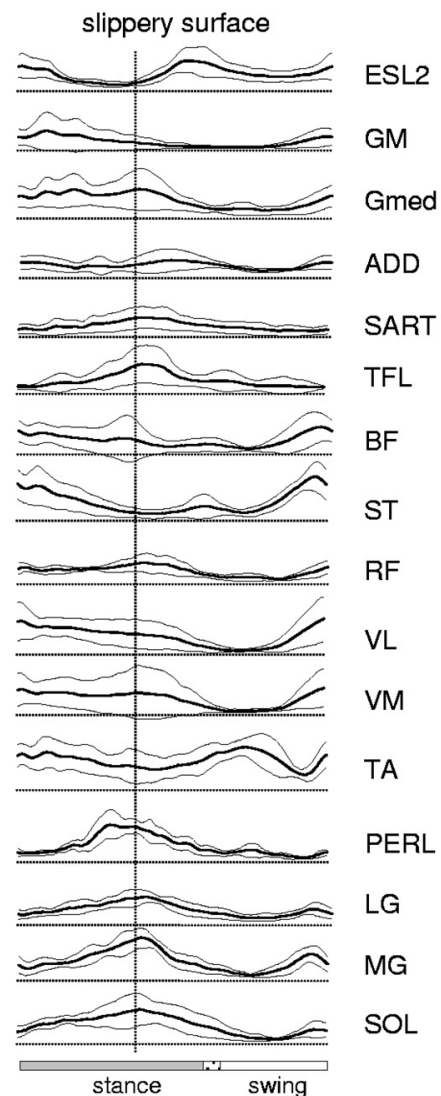

\section{C}

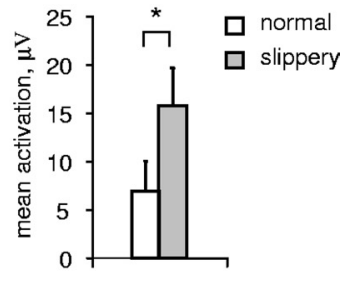

FIG. 9. EMG patterns. A: ensemble-averaged $( \pm \mathrm{SD})$ activity patterns of 16 ipsilateral muscles recorded from 6 subjects. Thin vertical dotted line indicates the approximate time of the peak activation of soleus (SOL), gastrocnemius medialis (MG), and gastrocnemius lateralis (LG) muscles. $B$ : corresponding spatiotemporal patterns of $\alpha$-motoneurons (MNs) in the lumbosacral enlargement. Output pattern for each segment was reconstructed by mapping the recorded EMG waveforms (nonnormalized method; Ivanenko et al. 2006) onto the known charts of segmental localization. Pattern is plotted vs. normalized gait cycle in a color scale using a filled contour plot. The black curves denote the instantaneous center of MN activity. $C$ : the mean activation level of the lumbosacral enlargement was about twofold higher on the slippery surface.

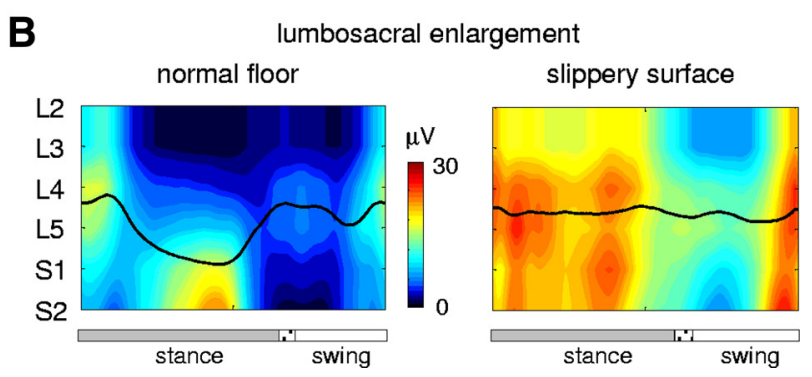

Foot motion in the sagittal plane was characterized by a single-peak elliptic trajectory during swing, resembling that of a nonplantigrade gait of toddlers (Forssberg 1985; Ivanenko et al. 2004). Indeed, greater toe grip, gentler heel strike, and a flatter shoe-floor angle at heel strike are strategies that have been shown as an adaptation to slippery surface (Fong et al. 2008b; Menant et al. 2009). The foot yaw angle oscillated much less, but foot slipping in the lateral direction consistently occurred during stance (Fig. 4) and implied a specific mediolateral balance strategy. Indeed, our results clearly demonstrated that walking on the slippery surface engaged systematic lateral trunk tilts in all subjects (Fig. 5).

The muscle activation patterns are also different when walking on normal and slippery surfaces and the difference persists unchanged throughout all trials (Table 2). Nevertheless, the timing of activity peaks relative to kinematics events is similar in the two conditions. Both activity patterns consist of distinct bursts of activity with a similar relationship to lift-off and touch-down events (Fig. 9). Specifically, the synergies involv- ing the alternating activation of proximal and distal limb muscles seen in normal walking are absent in slippery-surface walking. Instead, different muscle synergies, some of which are never observed in normal walking, are consistently seen in slippery-surface walking (Fig. 10).

We have reported this is the type of relationship between activation timing and muscle synergies for other walking conditions (Ivanenko et al. 2005, 2008). The timing bursts are consistent across tasks, although the muscles that are activated by each timed burst are task dependent. We suggested that this pattern is consistent with a neuronal network in which the timing of activity generated by CPG neurons is directed to the motoneurons via a premotor network that distributes the activity to motoneurons in a task-dependent manner determined by sensory and descending control information (Giszter et al. 2007; Ivanenko et al. 2009).

All of these gait parameters changed from those seen in normal walking within a single step (Table 1) as in the gait shift that occurs from walking to running (Cappellini et al. 

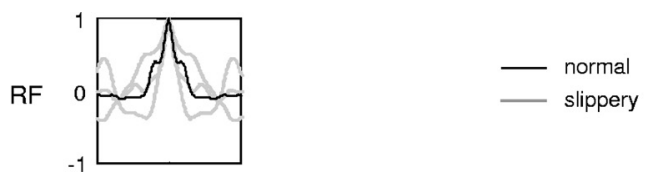
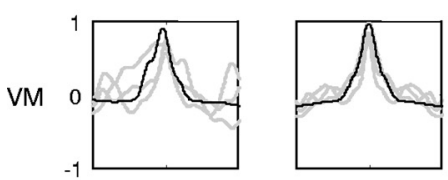

VL
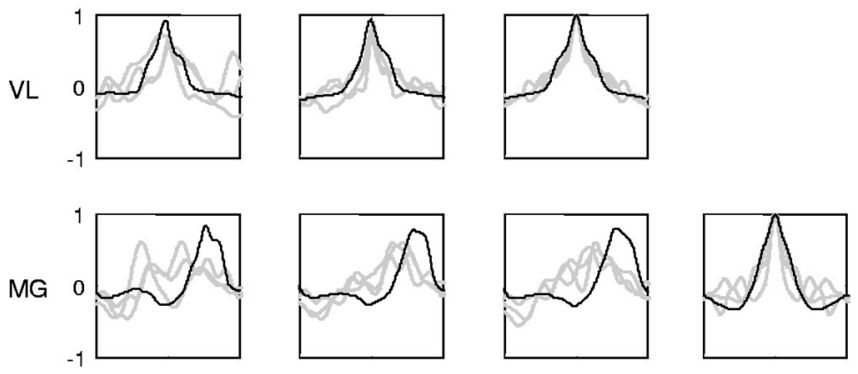

LG
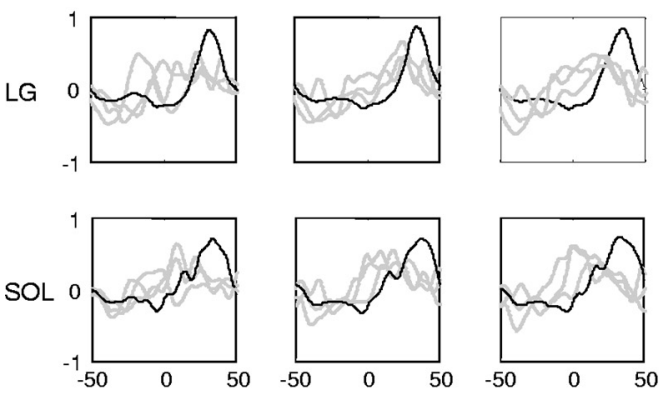

VM

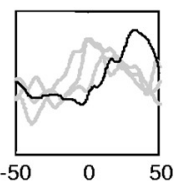

VL
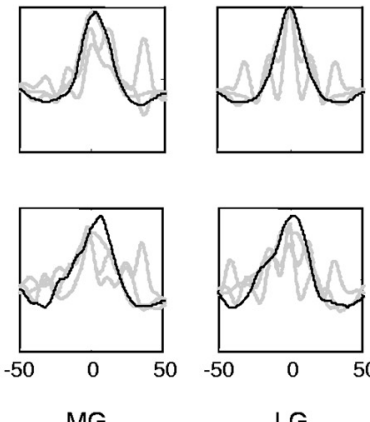

MG

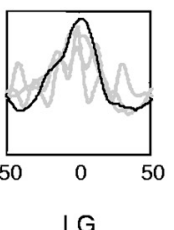

LG
FIG. 10. Cross-correlations between pairs of EMGs for the 2 synergistic groups of extensor muscles (RF, VL, VM, LG, MG, and SOL) from one subject walking on the normal floor and slippery surface at the same speed $(2.8 \mathrm{~km} / \mathrm{h})$. Each curve corresponds to one individual cycle. One representative cycle is illustrated for normal floor condition (black curves) and 3 cycles for slippery surface condition (superimposed gray curves). Cross-correlation values are plotted as a function of time delay (expressed as percentage of the gait cycle). A positive value of crosscorrelation at a negative time delay indicates that the activation of the column muscle leads that of the row muscle, whereas a positive crosscorrelation at a positive delay indicates a time lag of the column muscle relative to the row muscle. Autocorrelations of the indicated muscles are on the diagonal. RF, rectus femoris; VL, vastus lateralis; VM, vastus medialis; LG, gastocnemius lateralis; MG, gastrocnemius medialis; SOL, soleus.

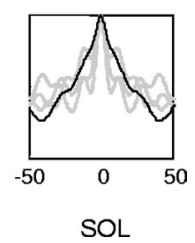

2006; Hreljac 1993; Nilsson et al. 1985). All the subjects showed comparable kinematic and EMG patterns immediately in the first trial and these patterns were no different for the final trial on the slippery surface (Tables 1 and 2). Therefore these results are all consistent with a change in gait mode rather than with an adaptation to existing surface conditions.

\section{Adaptations to the slippery surface}

Although the gait parameters were generally stable throughout the trials on the slippery surface, some differences were noted between the first and last trials. The most significant change to occur with repeated trials was in the amplitude of lateral hip and COM oscillations (Fig. 7, Table 1). In the first trial the COM displacements were similar to those of normal walking (Fig. 7), suggesting that smaller hip (and COM) lateral oscillations are not a trivial result of physics (low-friction forces) but depend on an appropriate bilateral coordination that leads to a lateral stabilization of the pelvis as a kind of reference platform (Fig. 5A). These changes in body kinematics may represent an adaptive modification of gait stability in response to the specific surface conditions in this task.

We suggest that locomotion can be adapted to actual surface conditions but that adaptation is separate from the gait change. Instead the gait change may occur when the slippery condition is expected to occur in the immediate future but not yet experienced.

\section{Anticipation of unstable surface conditions}

This expectation effect has also been observed previously by Cham and Redfern (2002) and Heiden et al. (2006) who reported that the perception of a potential risk of slipping leads to changes in the kinematics and kinetics of gait even when the slips do not occur. The changes seem to reflect a gait strategy that tends to keep the COM centered over the supporting limb and also increases limb stiffness and it may therefore be well suited to uncertain surface conditions in general. Thus one might expect to see a similar gait mode adopted on rugged, irregular terrain or on a ship tossing at sea. If so, it might reflect a cognitive influence on locomotor pattern generation that in each case could respond further to specific perturbations.

In the case of the slippery surface, our subjects were initially told that it was slippery and that they might be in danger of falling, but they did not know the specific surface conditions. The gait strategy they first adopted helped to prevent falls but it did not compensate fully for the low coefficient of friction, particularly in the lateral direction (Fig. 2A). Thus it was necessary to compensate for the lateral slips by adapting the control to optimize the lateral shear forces. 
The idea that there is a specific gait mode for uncertain surface conditions may also have clinical implications. For example, we conjecture that this gait might be used when the perception of the surface is uncertain, given that it might occur with sensory neuropathies or central nervous disorders like Parkinson's disease (PD) that may compromise proprioception (Konczak et al. 2009). The motor disorder that defines PD leads to a characteristic gait mode in which there is a bent posture and a nonplantigrade gait (Kimmeskamp and Henning 2001), with small steps and minimal joint rotations (Grasso et al. 1999). These patients also have decreased step length and a slower pace (Murray et al. 1978). Interestingly, they have also been reported to show considerable overlap in the activation of VL and gastrocnemius muscles, as we observed for slippery walking (Mitoma et al. 2000).

\section{A}

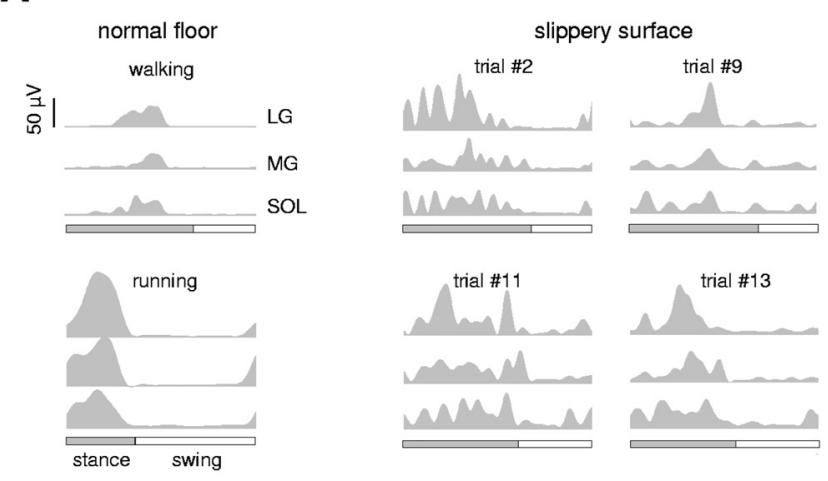

B timing of the main peak of calf muscles
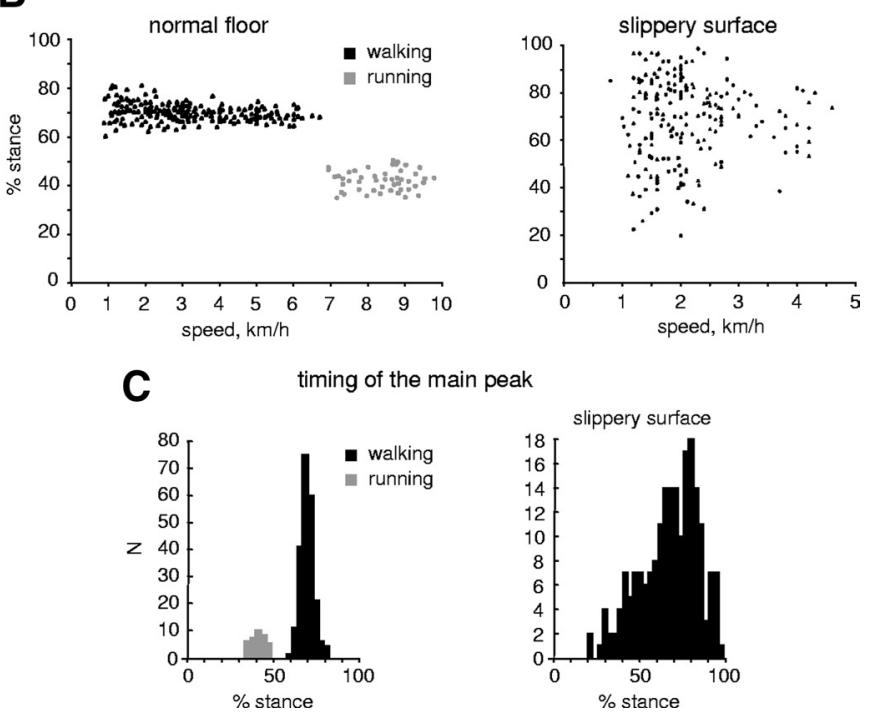

FIG. 11. Activation of distal extensors (LG, MG, and SOL) in different gaits. A: examples of individual cycle activity during normal walking and running (left) and walking on the slippery surface in one representative subject. The EMG records were numerically rectified and low-pass filtered with a cutoff of $10 \mathrm{~Hz}$. Note multiple bursts and a partial divergence of activity of LG, $\mathrm{MF}$, and SOL on the slippery surface. $B$ : relative timing (in percentage of stance) of the main peak of activity of posterior calf muscles (LG, MG, and SOL activities were averaged for this analysis) in different gaits as a function of speed. The data of all steps in all subjects are presented. Each point represents one individual cycle. $C$ : the corresponding distribution of the timing of the main peak of activity of calf muscles in different gaits. The data for all speeds were collapsed.
TABLE 2. EMG waveforms for each muscle were averaged across steps for each trial of walking on the slippery surface and for matched-speed trials on the normal surface

\begin{tabular}{lcc}
\hline \hline \multicolumn{1}{c}{ Event } & First Step First Trial & First Step Last Trial \\
\hline Trial 1 & $0.79 \pm 0.07$ & $0.82 \pm 0.04$ \\
Trial 2 & $0.76 \pm 0.09$ & $0.80 \pm 0.05$ \\
Trial 3 & $0.75 \pm 0.06$ & $0.76 \pm 0.04$ \\
Trial 4 & $0.72 \pm 0.13$ & $0.77 \pm 0.04$ \\
Trial 5 & $0.78 \pm 0.08$ & $0.78 \pm 0.03$ \\
Trial 6 & $0.85 \pm 0.04$ & $0.80 \pm 0.04$ \\
Trial 7 & $0.82 \pm 0.08$ & $0.78 \pm 0.07$ \\
Trial 8 & $0.81 \pm 0.04$ & $0.79 \pm 0.09$ \\
Trial 9 & $0.82 \pm 0.05$ & $0.78 \pm 0.03$ \\
Trial 10 & $0.79 \pm 0.06$ & $0.78 \pm 0.06$ \\
Trial 11 & $0.80 \pm 0.05$ & $0.77 \pm 0.06$ \\
Trial 12 & $0.83 \pm 0.05$ & $0.84 \pm 0.08$ \\
Trial 13 & $0.82 \pm 0.06$ & $0.84 \pm 0.07$ \\
Normal walking & $0.39 \pm 0.12$ & $0.32 \pm 0.23$ \\
\hline
\end{tabular}

Trials were each correlated with the EMG waveforms for either the first step in the first trial or the first step in the last trial.

In the case of peripheral neuropathies, patients tend to adopt a "conservative gait pattern" with less ankle mobility, slower walking speeds, longer stance phases, and smaller joint angle excursions than those of control subjects (e.g., Mueller et al. 1994). Subjects also show more cocontractions of agonist and antagonist muscles at the ankle and knee joints during stance phase compared with those of control subjects, once again comparable to slippery walking (Kwon et al. 2003).

Toddlers often adopt a similar conservative gait strategy having a nonplatigrade gait with a single peak foot lift during swing (see also Fig. 4A; Dominici et al. 2007; Forssberg 1985). This has been considered as an optimal strategy given the underdeveloped internal representations of both the support surface and precise foot position in space.

These above-cited findings suggest that subjects may adopt a gait mode not unlike that seen on slippery surfaces whenever there is a major uncertainty about the walking surface, even when that uncertainty may be due to a lack of sensory information or an inability to adequately interpret the surface characteristics.

\section{Conclusion}

In summary, walking on a slippery surface evokes a gait that is distinctly different from that used during normal walking. It seems to reflect a strategy for dealing with uncertain surface conditions. The strategy specifically reduces the horizontal forces while maintaining vertical forces. Thus the limb acts more like a strut providing primarily the vertical contact forces. The general increase in MN excitability evidenced by the general increase in MN activity (Chamber and Cham 2007; Heiden et al. 2006) may also correspond to a greater responsiveness to sensory inputs. Therefore the strategy may also increase the sensitivity to perturbations caused by surface conditions. As we noted, however, it may also serve as a conservative strategy when sensory information is unavailable.

\section{G R A N T S}

This work was supported by the Italian Health Ministry, Italian University Ministry (Programmi di ricerca di Rilevante Interesse Nazionale), and Disturbi 
del Controllo Motorio e Cardiorespiratorio grant from the Italian Space Agency.

\section{R E F E R E N C E S}

Alexander RM. Optimization and gaits in the locomotion of vertebrates. Physiol Rev 69: 1199-1227, 1989.

Barliya A, Omlor L, Giese MA, Flash T. An analytical formulation of the law of intersegmental coordination during human locomotion. Exp Brain Res 193: 371-385, 2009.

Bhatt T, Pai YC. Can observational training substitute motor training in preventing backward balance loss after an unexpected slip during walking? J Neurophysiol 99: 843-852, 2008.

Bhatt T, Pai YC. Generalization of gait adaptation for fall prevention: from moveable platform to slippery floor. J Neurophysiol 101: 948-957, 2009.

Bianchi L, Angelini D, Lacquaniti F. Individual characteristics of human walking mechanics. Pflügers Arch 436: 343-356, 1998.

Borghese NA, Bianchi L, Lacquaniti F. Kinematic determinants of human locomotion. J Physiol 494: 863-879, 1996.

Bosco G, Eian J, Poppele RE. Phase-specific sensory representations in spinocerebellar activity during stepping: evidence for a hybrid kinematic/ kinetic framework. Exp Brain Res 175: 83-96, 2006.

Brady RA, Pavol MJ, Owings TM, Grabiner MD. Foot displacement but not velocity predicts the outcome of a slip induced in young subjects while walking. J Biomech 33: 803-808, 2000.

Bresler E, Frankel JB. The forces and moments in the leg during level walking. J Appl Mech 72: 27-36, 1950.

Cappellini G, Ivanenko YP, Poppele RE, Lacquaniti F. Motor patterns in human walking and running. J Neurophysiol 95: 3426-3437, 2006.

Cham R, Redfern MS. Changes in gait when anticipating slippery floors. Gait Posture 15: 159-171, 2002.

Chambers AJ, Cham R. Slip-related muscle activation patterns in the stance leg during walking. Gait Posture 25: 565-572, 2007.

Clarac F, Brocard F, Vinay L. The maturation of locomotor networks. Prog Brain Res 143: 57-66, 2004.

Collins JJ. Gait transitions. In: The Handbook of Brain Theory and Neural Networks (2nd ed.), edited by Arbib MA. Cambridge, MA: The MIT Press, 2003, p. 463-466.

Courtine G, Schieppati M. Human walking along a curved path. II. Gait features and EMG patterns. Eur J Neurosci 18: 191-205, 2003.

Courtine G, Schieppati M. Tuning of a basic coordination pattern constructs straight-ahead and curved walking in humans. J Neurophysiol 91: 15241535, 2004.

Cuellar CA, Tapia JA, Juarez V, Quevedo J, Linares P, Martinez L, Manjarrez E. Propagation of sinusoidal electrical waves along the spinal cord during a fictive motor task. J Neurosci 29: 798-810, 2009.

Day SJ, Hulliger M. Experimental simulation of cat electromyogram: evidence for algebraic summation of motor-unit action-potential trains. $\mathrm{J} \mathrm{Neu}$ rophysiol 86: 2144-2158, 2001.

Dominici N, Ivanenko YP, Lacquaniti F. Control of foot trajectory in walking toddlers: adaptation to load changes. J Neurophysiol 97: 27902801, 2007.

Fong DT, Hong Y, Li JX. Lower extremity preventive measures for slips: joint moments and myoelectric analysis. Ergonomics 51: 1830-1846, 2008a.

Fong DT, Mao DW, Li JX, Hong Y. Greater toe grip and gentler heel strike are the strategies to adapt to slippery surface. J Biomech 41: 838-844, 2008 b.

Forssberg H. Ontogeny of human locomotor control. I. Infant stepping, supported locomotion and transition to independent locomotion. Exp Brain Res 57: 480-493, 1985.

Full RJ, Koditschek DE. Templates and anchors: neuromechanical hypotheses of legged locomotion on land. J Exp Biol 202: 3325-3332, 1999.

Giszter S, Patil V, Hart C. Primitives, premotor drives, and pattern generation: a combined computational and neuroethological perspective. Prog Brain Res 165: 323-346, 2007.

Golubitsky M, Stewart I, Buono PL, Collins JJ. Symmetry in locomotor central pattern generators and animal gaits. Nature 401: 693-695, 1999.

Grasso R, Bianchi L, Lacquaniti F. Motor patterns for human gait: backward versus forward locomotion. J Neurophysiol 80: 1868-1885, 1998.

Grasso R, Ivanenko YP, Zago M, Molinari M, Scivoletto G, Castellano V, Macellari V, Lacquaniti F. Distributed plasticity of locomotor pattern generators in spinal cord injured patients. Brain 127: 1019-1034, 2004.

Grasso R, Peppe A, Stratta F, Angelini D, Zago M, Stanzione P, Lacquaniti F. Basal ganglia and gait control: apomorphine administration and internal pallidum stimulation in Parkinson's disease. Exp Brain Res 126: 139-148, 1999.

Grasso R, Zago M, Lacquaniti F. Interactions between posture and locomotion: motor patterns in humans walking with bent posture versus erect posture. J Neurophysiol 83: 288-300, 2000.

Grillner S. Control of locomotion in bipeds, tetrapods, and fish. In: Handbook of Physiology. The Nervous System. Motor Control. Bethesda, MD: Am. Physiol. Soc., 1981, sect. 1, vol. II, pt. 2, p. 1179-1236.

Grönqvist R, Hirvonen M, Tuusa A. Slipperiness of the shoe-floor interface: comparison of objective and subjective assessments. Appl Ergonomics 24: 258-262, 1993.

Heiden TL, Sanderson DJ, Inglis JT, Siegmund GP. Adaptations to normal human gait on potentially slippery surfaces: the effects of awareness and prior slip experience. Gait Posture 24: 237-246, 2006.

Hirasaki E, Moore ST, Raphan T, Cohen B. Effects of walking velocity on vertical head and body movements during locomotion. Exp Brain Res 127: 117-130, 1999.

Hoffer JA, Sugano N, Loeb GE, Marks WB, O'Donovan MJ, Pratt CA. Cat hindlimb motoneurons during locomotion. II. Normal activity patterns. J Neurophysiol 57: 530-553, 1987.

Hreljac A. Preferred and energetically optimal gait transition speeds in human locomotion. Med Sci Sports Exerc 25: 1158-1162, 1993.

Ivanenko YP, Cappellini G, Dominici N, Poppele RE, Lacquaniti F. Coordination of locomotion with voluntary movements in humans. $\mathrm{J} \mathrm{Neu}$ rosci 25: 7238-7253, 2005.

Ivanenko YP, Cappellini G, Dominici N, Poppele RE, Lacquaniti F. Modular control of limb movements during human locomotion. J Neurosci 27: 11149-11161, 2007.

Ivanenko YP, Cappellini G, Poppele RE, Lacquaniti F. Spatiotemporal organization of alpha-motoneuron activity in the human spinal cord during different gaits and gait transitions. Eur J Neurosci 27: 3351-3368, 2008.

Ivanenko YP, Dominici N, Cappellini G, Dan B, Cheron G, Lacquaniti F. Development of pendulum mechanism and kinematic coordination from the first unsupported steps in toddlers. J Exp Biol 207: 3797-3810, 2004.

Ivanenko YP, Grasso R, Macellari V, Lacquaniti F. Control of foot trajectory in human locomotion: role of ground contact forces in simulated reduced gravity. J Neurophysiol 87: 3070-3089, 2002.

Ivanenko YP, Poppele RE, Lacquaniti F. Spinal cord maps of spatiotemporal alpha-motoneuron activation in humans walking at different speeds. J Neurophysiol 95: 602-618, 2006.

Ivanenko YP, Poppele RE, Lacquaniti F. Distributed neural networks for controlling human locomotion: lessons from normal and SCI subjects. Brain Res Bull 78: 13-21, 2009.

Kajita S, Kaneko K, Harada K, Kanehiro F, Fujiwara K, Hirukawa H. Biped walking on a low friction floor. In: Proceedings of 2004 IEEE/ RSJ International Conference on Intelligent Robots and Systems. Piscataway, NJ: IEEE, 2004, p. 3546-3552.

Kendall FP, McCreary EK, Provance PG. Muscles: Testing and Function. Baltimore, MD: Williams \& Wilkins, 1993.

Kimmeskamp S, Hennig EM. Heel to toe motion characteristics in Parkinson patients during free walking. Clin Biomech (Bristol, Avon) 16: 806-812, 2001

Konczak J, Corcos DM, Horak F, Poizner H, Shapiro M, Tuite P, Volkmann J, Maschke M. Proprioception and motor control in Parkinson's disease. J Mot Behav 10: 1-11, 2009.

Kuo AD, Donelan JM, Ruina A. Energetic consequences of walking like an inverted pendulum: step-to-step transitions. Exerc Sport Sci Rev 33: 88-97, 2005.

Kwon OY, Minor SD, Maluf KS, Mueller MJ. Comparison of muscle activity during walking in subjects with and without diabetic neuropathy. Gait Posture 18: 105-113, 2003.

Lacquaniti F, Grasso R, Zago M. Motor patterns in walking. News Physiol Sci 14: 168-174, 1999.

Lacquaniti F, Ivanenko YP, Zago M. Kinematic control of walking. Arch Ital Biol 140: 263-272, 2002.

Lockhart TE, Kim S. Relationship between hamstring activation rate and heel contact velocity: factors influencing age-related slip-induced falls. Gait Posture 24: 23-34, 2006.

Lockhart TE, Spaulding JM, Park SH. Age-related slip avoidance strategy while walking over a known slippery floor surface. Gait Posture 26: 142-149, 2007.

Margaria R. Biomechanics and Energetics of Muscular Exercise. Oxford, UK: Clarendon Press, 1976 
Marigold DS, Patla AE. Strategies for dynamic stability during locomotion on a slippery surface: effects of prior experience and knowledge. J Neurophysiol 88: 339-353, 2002.

Marsh RL, Ellerby DJ, Carr JA, Henry HT, Buchanan CI. Partitioning the energetics of walking and running: swinging the limbs is expensive. Science 303: $80-83,2004$.

Menant JC, Steele JR, Menz HB, Munro BJ, Lord SR. Effects of walking surfaces and footwear on temporo-spatial gait parameters in young and older people. Gait Posture 29: 392-397, 2009.

Mentel T, Cangiano L, Grillner S, Buschges A. Neuronal substrates for state-dependent changes in coordination between motoneuron pools during fictive locomotion in the lamprey spinal cord. J Neurosci 28: 868-879, 2008.

Menz HB, Lord SR, Fitzpatrick RC. Acceleration patterns of the head and pelvis when walking on level and irregular surfaces. Gait Posture 18: 35-46, 2003.

Mitoma H, Hayashi R, Yanagisawa N, Tsukagoshi H. Characteristics of parkinsonian and ataxic gaits: a study using surface electromyograms, angular displacements and floor reaction forces. J Neurol Sci 174: 22-39, 2000.

Moyer BE, Redfern MS, Cham R. Biomechanics of trailing leg response to slipping: evidence of interlimb and intralimb coordination. Gait Posture 29: 565-570, 2009.

Mueller MJ, Minor SD, Sahrmann SA, Schaaf JA, Strube MJ. Differences in the gait characteristics of patients with diabetes and peripheral neuropathy compared with age-matched controls. Phys Ther 74: 299-308, 1994.

Murray MP, Sepic SB, Gardner GM, Downs WJ. Walking patterns of men with parkinsonism. Am J Phys Med 57: 278-294, 1978.

Nilsson J, Thorstensson A, Halbertsma J. Changes in leg movements and muscle activity with speed of locomotion and mode of progression in humans. Acta Physiol Scand 123: 457-475, 1985.

Oates AR, Frank JS, Patla AE, VanOoteghem K, Horak FB. Control of dynamic stability during gait termination on a slippery surface in Parkinson's disease. Mov Disord 23: 1977-1983, 2008.

Oates AR, Patla AE, Frank JS, Greig MA. Control of dynamic stability during gait termination on a slippery surface. J Neurophysiol 93: 64-70, 2005 .

Olree KS, Vaughan CL. Fundamental patterns of bilateral muscle activity in human locomotion. Biol Cybern 73: 409-414, 1995.

Park JH, Kwon O. Reflex control of biped robot locomotion on a slippery surface. In: Proceedings of the 2001 IEEE International Conference on Robotics and Automation. Piscataway, NJ: IEEE, 2001, p. 4134-4139.

Pepin A, Norman KE, Barbeau H. Treadmill walking in incomplete spinalcord-injured subjects: 1. Adaptation to changes in speed. Spinal Cord 41: 257-270, 2003.

Perkins PJ. Measurement of slip between the shoe and ground during walking. In: Walkway Surfaces: Measurement of Slip Resistance (ASTM Special Technical Publication 649-EB), edited by Anderson C, Senne J. Philadelphia, PA: American Society for Testing and Materials (ASTM), 1978, p. 71-87.

Phillips LH 2nd, Park TS. Electrophysiologic mapping of the segmental anatomy of the muscles of the lower extremity. Muscle Nerve 14: 1213$1218,1991$.
Poppele R, Bosco G. Sophisticated spinal contributions to motor control. Trends Neurosci 26: 269-276, 2003.

Pozzo T, Berthoz A, Lefort L. Head stabilization during various locomotor tasks in humans. I. Normal subjects. Exp Brain Res 82: 97-106, 1990.

Redfern MS, Cham R, Gielo-Perczak K, Grönqvist R, Hirvonen M, Lanshammar H, Marpet M, Pai CY, Powers C. Biomechanics of slips. Ergonomics 44: 1138-1166, 2001.

Saibene F, Minetti AE. Biomechanical and physiological aspects of legged locomotion in humans. Eur J Appl Physiol 88: 297-316, 2003.

Sharrard WJW. The distribution of the permanent paralysis in the lower limb in poliomyelitis. J Bone Joint Surg 37: 540-558, 1955.

Sharrard WJW. The segmental innervation of the lower limb muscles in man. Ann R Coll Surg Engl 35: 106-122, 1964.

Srinivasan M, Ruina A. Computer optimization of a minimal biped model discovers walking and running. Nature 439: 72-75, 2006

Stewart JD. Electrophysiological mapping of the segmental anatomy of the muscles of the lower extremity. Muscle Nerve 15: 965-966, 1992.

Strandberg L. On accident analysis and slip-resistance measurement. Ergonomics 26: 11-32, 1983.

Strandberg L, Lanshammar H. The dynamics of slipping accidents. J Occup Accidents 3: 153-162, 1981.

Tang PF, Woollacott MH, Chong RK. Control of reactive balance adjustments in perturbed human walking: roles of proximal and distal postural muscle activity. Exp Brain Res 119: 141-152, 1998.

Tesio L, Lanzi D, Detrembleur C. The 3-D motion of the centre of gravity of the human body during level walking. I. Normal subjects at low and intermediate walking speeds. Clin Biomech 13: 77-82, 1998.

Troy KL, Donovan SJ, Grabiner MD. Theoretical contribution of the upper extremities to reducing trunk extension following a laboratory-induced slip. J Biomech 42: 1339-1344, 2009.

Troy KL, Grabiner MD. Recovery responses to surrogate slipping tasks differ from responses to actual slips. Gait Posture 24: 441-447, 2006.

Wakeling JM, Horn T. Neuromechanics of muscle synergies during cycling. J Neurophysiol 101: 843-854, 2009.

Winter DA. The Biomechanics and Motor Control of Human Gait: Normal, Elderly and Pathological. Waterloo, Ontario, Canada: Waterloo Biomechanics Press, 1991.

Yakovenko S, Mushahwar V, VanderHorst V, Holstege G, Prochazka A. Spatiotemporal activation of lumbosacral motoneurons in the locomotor step cycle. J Neurophysiol 87: 1542-1553, 2002.

Yang F, Anderson FC, Pai YC. Predicted threshold against backward balance loss following a slip in gait. J Biomech 41: 1823-1831, 2008.

You J, Chou Y, Lin C, Su F. Effect of slip on movement of body center of mass relative to base of support. Clin Biomech (Bristol, Avon) 16: 167-173, 2001.

Zatsiorsky V, Seluyanov V, Chugunova L. In vivo body segment inertial parameters determination using a gamma-scanner method. In: Biomechanics of Human Movement: Applications in Rehabilitation, Sports and Ergonomics, edited by Berme N, Cappozzo A. Worthington, OH: Bertec, 1990, p. $186-202$ 